\title{
20. RADIOLARIAN RESPONSE TO OCEANOGRAPHIC CHANGES IN THE EASTERN EQUATORIAL PACIFIC AT 2.3 AND 4.8 MA: RELATIONSHIP BETWEEN CHANGING CARBONATE DEPOSITION AND SURFACE OCEANOGRAPHY ${ }^{1}$
}

\author{
Nicklas G. Pisias ${ }^{2}$ and Theodore C. Moore, $\mathrm{Jr}^{3}$
}

\begin{abstract}
Two short time intervals centered at 2.3 and $4.7 \mathrm{Ma}$ were studied to investigate short-term variations in surface-ocean processes as indicated by changes in the radiolarian microfossil population. These time intervals represent two different settings of late Neogene climate. The older interval represents a time when tropical circulation between the Pacific and Atlantic oceans was not blocked by the Isthmus of Panama, whereas the younger interval represents a time when Northern Hemisphere glaciation was present but did not display the dominance of the 100,000-yr cycle that characterizes the late Pleistocene. The younger time slice at 2.3 Ma was sampled at all Leg 138 sites except Site 844, where significant reworking was evident. All sites except 844, 853, and 854 were sampled for the older time slice. Samples were taken at 10- to $20-\mathrm{cm}$ intervals at each site and spanned a GRAPE density maximum and minimum. Thus, it was possible to investigate whether the changes in carbonate content (as indicated by GRAPE density) were associated with changes in surface-ocean conditions (indicated by radiolarian assemblage variations).

For both time slices, the radiolarian data indicate that intervals of decreased carbonate content are periods of cooler water conditions and possibly enhanced biogenic production. Times of increased carbonate content are associated with inferred warmer oceanographic conditions, as indicated by the dominance of tropical assemblages at $2.3 \mathrm{Ma}$ and tropical and western Pacific assemblages during the time slice centered at $4.8 \mathrm{Ma}$. However, the spatial patterns of change during each time slice show a distinct difference in the mapped patterns of radiolarian assemblage dominance. The older time slice, representing a period before the closing of the Isthmus of Panama, shows more zonal patterns presumably associated with a more zonal character of equatorial circulation. After the closing of the isthmus, the shifts in faunal patterns between times of high and low carbonates are characterized by shifts in the dominance of the tropical and transitional assemblages, respectively, throughout the region.
\end{abstract}

\section{INTRODUCTION}

One of the remarkable features of the sediment sections collected during Ocean Drilling Program (ODP) Leg 138 is the rhythmic pattern of variations in calcium carbonate concentration. These patterns can be correlated over a significant region of the eastern equatorial Pacific (Shackleton et al., 1992) and can be used to define a detailed, orbitally derived time scale (Shackleton et al., this volume). Together, drill Sites 846 through 853 provide a high-resolution record of eastern equatorial Pacific sediment variations spanning the last 10 m.y.

Shackleton et al. (this volume) show that a very high degree of coherence (correlation) exists between solar insolation changes and the GRAPE density records, which in these sites provide an excellent proxy for calcium carbonate content (Hagelberg et al., this volume). Variations in carbonate content reflect the interplay between surface production of calcium carbonate, dissolution and dilution by noncarbonate sediments. The variations in solar radiation are the result of the rhythmic changes in the tilt and precession of the earth's axis. The insolation record used by Shackleton et al. (this volume) was for summer insolation at $65^{\circ} \mathrm{N}$. This record was selected because of its relative contributions of tilt and precessional components, not because a causal mechanism is directly related to summer insolation at high northern latitudes. One of the fundamental questions related to the study of climate change on time scales related to orbital forcing is, what are the linking mechanisms between insolation and climate response? Why, for example, do carbonate concentration variations in the equatorial Pacific Ocean contain such a strong insolation response?

${ }^{1}$ Pisias, N.G., Mayer, L.A., Janecek, T.R., Palmer-Julson, A., and van Andel, T.H (Eds.), 1995. Proc. ODP, Sci. Results, 138: College Station, TX (Ocean Drilling Program)

${ }^{2}$ College of Oceanic and Atmospheric Sciences, Oregon State University, Oceanography Administration Building 104, Corvallis, OR 97331-5503, U.S.A.

${ }^{3}$ Center for Great Lakes and Aquatic Sciences, University of Michigan, Ann Arbor, MI 48109-2099, U.S.A.
Detailed analysis of the data sets spanning the last 6 m.y. by Hagelberg et al. (this volume) demonstrates that two distinct modes of variation can be extracted from proxy carbonate records of these sites. The spatial patterns of these modes of variation led Hagelberg et al. to conclude that variability in surface-ocean processes (i.e., upwelling, advection, etc.) play an important role in controlling the observed variability in calcium carbonate content of eastern equatorial Pacific sediments.

The purpose of this study is to provide direct evidence on how surface-ocean processes may have changed in association with changes in carbonate deposition. Arrhenius (1952) first proposed that the variations in calcium carbonate in late Pleistocene sediments of the equatorial Pacific reflected changes in near-surface biologic productivity in response to glacial-interglacial climate changes. Later workers argued that dissolution of carbonate better explained these carbonate cycles (e.g., Berger, 1973), though some noted that surface production must also be important (Adelseck and Anderson, 1978). More recently, Farrell and Prell $(1989,1991)$ presented a compilation of central equatorial Pacific sediment records and concluded that, although little change occurred in the calcite compensation depth during the late Neogene, significant changes in the depth of the calcite lysocline could be documented and thus carbonate variations in the central part of the equatorial Pacific were related to dissolution changes. Archer (1991a, 1991b) questioned this conclusion of Farrell and Prell (1989) and, based on model calculations, suggested that production changes may have been important in controlling carbonate variations.

\section{STRATEGY AND OBJECTIVES}

In this study, we use radiolarian species census data to infer surface oceanographic changes. Ideally, micropaleontologic data at the same resolution as the carbonate proxy data could be used to determine the degree of spatial and temporal coherence of these surfaceocean indicators. In this way, we could hope to provide an initial test of the hypothesis of Hagelberg et al. (this volume). Recognizing that 
this data acquisition task was beyond the scope of a post-cruise research project, we designed a much-reduced sampling strategy to provide initial insights about the following paleoceanographic questions:

1. Are marked changes in the carbonate content of sediments associated with changes in surface-ocean indicators?

2 . Is the relationship between high and low carbonate contents and surface indicators of climatic and oceanographic states constant with time?

To begin to address these questions, we sampled and completed radiolarian species census on two time slices centered at about $2.3 \mathrm{Ma}$ and at about $4.8 \mathrm{Ma}$. In addition to the radiolarian census data for these two time slices, we have radiolarian data from surface sediments of the Pacific basin (N.G. Pisias and A.C. Mix, unpubl. data).

Each of these time slices spans an interval of high and low carbonate concentrations and allows us to examine the nature of surface oceanographic variability during what are presumed to be two different climate regimes as compared to data spanning the late Pleistocene. Compared to the late Pleistocene, these two times are oceanographically and climatically quite different. The oldest interval is considered to be a time of open circulation between the tropical Pacific and Atlantic oceans through the Isthmus of Panama. The younger interval represents a time after the closure of the isthmus and near the beginning of Northern Hemisphere glaciation, when (unlike the late Pleistocene) little climatic variance was associated with 100,000 -yr cycles.

\section{METHODS}

\section{Samples}

Using the stratigraphic framework established during Leg 138 (Shackleton et al., 1992), sampling intervals were selected for each of the 11 Leg 138 sites. Because of evidence of significant reworking of the radiolarian fauna in Site 844, we do not present data from this site. The stratigraphic framework combines the distinct pattern of GRAPE density variations in Leg 138 sites with high-resolution biostratigraphy and magnetostratigraphy to define 20 correlative levels for Sites 846 through 853 . The identification of correlative intervals for Sites 845 and 854 is much more problematic. The sediments at these sites, for the most part, had little calcium carbonate during the late Neogene, and GRAPE density records contain much lower amplitude variations. Correlation in these sites relies much more heavily on biostratigraphic and magnetostratigraphic information.

Samples studied are listed in Table 1. For Time Slice 1, samples were taken to span a carbonate cycle starting with a carbonate low (GRAPE density minimum) and ending in a carbonate high (GRAPE density maximum). This carbonate cycle was easily seen during the sampling of each site by a distinct color change from dark (carbonate low) to a more white (carbonate high) sediment color. For the older time slice (Time Slice 2), samples were taken at two levels in each site. The older level, centered at about $4.87 \mathrm{Ma}$, was a distinct carbonate maximum, whereas the younger level, centered at about $4.75 \mathrm{Ma}$, was a marked carbonate minimum. These levels were selected on the basis of assuming that the distinct carbonate features could be more easily correlated from site to site. The GRAPE density records for the intervals sampled and the location of radiolarian samples are illustrated in Figure 1.

As part of post-cruise research, Shackleton et al. (this volume) developed a high-resolution chronology for Sites 846 through 852 using the strategy of orbital tuning.

Using these chronologies and chronologies based on paleomagnetic stratigraphy for Sites 845,853 , and 854 , we plot the GRAPE density records vs. age in Figure 2. Also shown are the samples taken as part of this study.

For Sites 846,848 , and 850 through 854 , the radiolarian samples span the interval from about 2.35 to about $2.4 \mathrm{Ma}$ and cross a GRAPE minimum and maximum at 2.38 and $2.36 \mathrm{Ma}$ (Fig. 2A). From Sites
847 and 849 , samples span the next youngest and oldest GRAPE minimum and maximum, respectively.

The GRAPE records and radiolarian samples for Time Slice 2 are plotted vs. the chronology of Shackleton et al. (this volume) in Figure 2B. For Sites 846 and 849 through 852 , radiolarian samples cover the GRAPE density maximum centered at around $4.86 \mathrm{Ma}$. The GRAPE density maximum sampled in Site 847 is estimated to be about 4.87 $\mathrm{Ma}$, and in Site 848 maxima were sampled at about 4.91 and $4.95 \mathrm{Ma}$. The GRAPE density minimum samples for Time Slice 2 are centered at about $4.75 \mathrm{Ma}$ in Sites 846 and 849 through 852 . At Site 848 , because of a sampling error, samples are available for an older GRAPE minimum at about 4.93 Ma. For Site 847, a GRAPE minimum at 4.83 Ma was sampled. For Site 845 , where the chronologic framework is of a lower resolution, samples are available for short intervals centered at 4.72 and 4.9 Ma. Radiolarians were not sufficiently preserved at Sites 853 and 854 for detailed analysis in Time Slice 2.

Thus, for the most part, comparisons of radiolarian fauna from the selected carbonate minima and maxima between sites appear to be time correlatable events based on the time scales developed by Shackleton et al. (this volume). It could be argued that the chronology for these sites could be further refined to provide a much higher correlation between GRAPE maxima and minima (e.g., can the GRAPE minimum at $2.38 \mathrm{Ma}$ in Site 846 be time correlatable to the $2.36-\mathrm{Ma}$ event in Site 847?). We have chosen not to make further refinements to the chronology of Shackleton et al. (this volume). Also, because of time and financial limitations, it was not possible to resample sites in which the original shipboard stratigraphy was of insufficient resolution to provide a complete, time-synchronous data set. However, we feel that this data set does provide sufficient resolution to begin to address the primary objectives set for this study.

\section{Radiolarian Faunal Analysis}

Samples were processed for radiolarian faunal analysis using the technique developed by Roelofs and Pisias (1986). Samples were first treated with $50 \%$ hydrochloric acid to remove calcium carbonate. Hydrogen peroxide and potassium dichromate were used to oxidize any organic carbon contained in the samples. Cleaned samples are sieved at $63 \mu \mathrm{m}$, and microscope slides were made by random settling of the $>63-\mu \mathrm{m}$ fraction onto microscope cover slips. At no time was an ultrasonic bath used to disperse samples. Quantitative species census data were collected by counting at least 800 radiolarian specimens per sample. Radiolarians were categorized into 92 counting groups (Table 2) plus a category for unidentified forms.

For analyzing these data, we used information about the distribution of extant radiolaria in surface sediments to provide an initial view of the oceanographic changes observed in the two time slices presented here. A database is now available that gives the distribution of radiolarians in surface sediments of the Pacific. Recent surface sediment distributions have been instrumental in our use of radiolarians to unravel past oceanographic change (e.g., Molina-Cruz, 1977; Moore, 1978; Romine and Moore, 1981; Schramm, 1985). Most of these studies concentrated on upper Pleistocene sediments, although few provided detailed quantitative radiolarian analyses for equatorial Pacific sections from the late Neogene (Alexandrovitch, 1989; Hays et al., 1989; Hagelberg and Pisias, 1990).

For a study of late Pleistocene aged sediment cores (N.G. Pisias and A.C. Mix, unpubl. data), paleoecological transfer functions were developed for the Pacific Ocean using the approach of Imbrie and Kipp (1971). This analysis was based on 170 surface sediment samples from the Pacific Basin. The advantage of this data set over other previously published analyses is that both the surface sediments and the sediments from the Leg 138 sites were counted using the same taxonomic framework.

Factor analysis was used to describe the surface data set in terms of seven factors. In the factor analysis, 41 species were ultimately retained. Details of the analysis are presented in N.G. Pisias and A.C. 
Table 1. Samples used in Time Slices 1 and 2.

\begin{tabular}{|c|c|c|c|c|c|c|c|c|}
\hline $\begin{array}{l}\text { Core, section, } \\
\text { interval }(\mathrm{cm})\end{array}$ & $\begin{array}{l}\text { Depth } \\
\text { (mbsf) }\end{array}$ & $\begin{array}{l}\text { Depth } \\
\text { (mcd) }\end{array}$ & $\begin{array}{l}\text { Core, section, } \\
\text { interval }(\mathrm{cm})\end{array}$ & $\begin{array}{l}\text { Depth } \\
\text { (mbsf) }\end{array}$ & $\begin{array}{l}\text { Depth } \\
\text { (mcd) }\end{array}$ & $\begin{array}{l}\text { Core, section, } \\
\text { interval }(\mathrm{cm})\end{array}$ & $\begin{array}{l}\text { Depth } \\
\text { (mbsf) }\end{array}$ & $\begin{array}{l}\text { Depth } \\
\text { (mcd) }\end{array}$ \\
\hline Time Slice 1: & & & $138-85 \mid \mathrm{C}-4 \mathrm{H}-5,63$ & 37.63 & 44.48 & $138-848 \mathrm{C}-5 \mathrm{H}-6,40$ & 41.90 & 46.45 \\
\hline $138-845 \mathrm{~B}-4 \mathrm{H}-6,125$ & 40.35 & 44.23 & $138-851 \mathrm{C}-4 \mathrm{H}-5,68$ & 37.68 & 44.53 & $138-848 \mathrm{C}-5 \mathrm{H}-6.50$ & 42.00 & 46.55 \\
\hline $138-845 \mathrm{~B}-4 \mathrm{H}-6,135$ & 40.45 & 44.33 & $138-851 \mathrm{C}-4 \mathrm{H}-5,73$ & 37.73 & 44.58 & $138-848 \mathrm{C}-5 \mathrm{H}-6,60$ & 42.10 & 46.65 \\
\hline I38-845B-4H-6, 145 & 40.55 & 44.43 & $138-85 \mid \mathrm{C}-4 \mathrm{H}-5,78$ & 37.78 & 44.63 & $138-848 \mathrm{C}-5 \mathrm{H}-6.70$ & 42.20 & 46.75 \\
\hline $138-845 \mathrm{~B}-4 \mathrm{H}-7,5$ & 40.65 & 44.53 & $138-851 \mathrm{C}-4 \mathrm{H}-5,83$ & 37.83 & 44.68 & $138-848 \mathrm{C}-5 \mathrm{H}-6,80$ & 42.30 & 46.85 \\
\hline $138-845 \mathrm{~B}-4 \mathrm{H}-7,15$ & 40.75 & 44.63 & $138-85 \mid \mathrm{C}-4 \mathrm{H}-5,88$ & 37.88 & 44.73 & $138-848 \mathrm{C}-5 \mathrm{H}-6,90$ & 42.40 & 46.95 \\
\hline $138-845 \mathrm{~B}-4 \mathrm{H}-7,25$ & 40.85 & 44.73 & $138-851 \mathrm{C}-4 \mathrm{H}-5,93$ & 37.93 & 44.78 & $138-848 \mathrm{C}-5 \mathrm{H}-6,100$ & 42.50 & 47.05 \\
\hline $138-845 \mathrm{~B}-4 \mathrm{H}-7,35$ & 40.95 & 44.83 & $|38-85| \mathrm{C}-4 \mathrm{H}-5,98$ & 37.98 & 44.83 & $138-848 \mathrm{C}-5 \mathrm{H}-6,105$ & 42.55 & 47.10 \\
\hline $138-846 \mathrm{D}-9 \mathrm{H}-1,77$ & 78.57 & 88.97 & $138-85$ IC-4H-5, 103 & 38.03 & 44.88 & $138-848 \mathrm{C}-5 \mathrm{H}-6.110$ & 42.60 & 47.15 \\
\hline 138-846D-9H-1, 87 & 78.67 & 89.07 & 138-85|C-4H-5, 108 & 38.08 & 44.93 & $138-848 \mathrm{C}-5 \mathrm{H}-6,115$ & 42.65 & 47.20 \\
\hline 138-846D-9H-I, 97 & 78.77 & 89.17 & $138-852 \mathrm{C}-4 \mathrm{H}-1,80$ & 25.80 & 27.00 & $138-848 \mathrm{C}-5 \mathrm{H}-6,120$ & 42.70 & 47.25 \\
\hline $138-846 \mathrm{D}-9 \mathrm{H}-1,107$ & 78.87 & 89.27 & $138-852 \mathrm{C}-4 \mathrm{H}-1,85$ & 25.85 & 27.05 & $138-848 \mathrm{C}-5 \mathrm{H}-6,125$ & 42.75 & 47.30 \\
\hline $138-846 \mathrm{D}-9 \mathrm{H}-1.117$ & 78.97 & 89.37 & $138-852 \mathrm{C}-4 \mathrm{H}-\mathrm{I}, 90$ & 25.90 & 27.10 & $138-848 \mathrm{C}-5 \mathrm{H}-6,130$ & 42.80 & 47.35 \\
\hline $138-846 \mathrm{D}-9 \mathrm{H}-\mathrm{I}, 127$ & 79.07 & 89.47 & $138-852 \mathrm{C}-4 \mathrm{H}-1,95$ & 25.95 & 27.15 & $138-848 \mathrm{C}-5 \mathrm{H}-6,135$ & 42.85 & 47.40 \\
\hline $138-846 \mathrm{D}-9 \mathrm{H}-1,137$ & 79.17 & 89.57 & $138-852 \mathrm{C}-4 \mathrm{H}-1,100$ & 26.00 & 27.20 & $138-848 \mathrm{C}-5 \mathrm{H}-6,140$ & 42.90 & 47.45 \\
\hline 138-846D-9H-1, 147 & 79.27 & 89.67 & $138-852 \mathrm{C}-4 \mathrm{H}-1,105$ & 26.05 & 27.25 & $138-848 \mathrm{C}-5 \mathrm{H}-6,145$ & 42.95 & 47.50 \\
\hline $138-846 \mathrm{D}-9 \mathrm{H}-2,7$ & 79.37 & 89.77 & $=138-852 \mathrm{C}-4 \mathrm{H}-1,110$ & 26.10 & 27.30 & 138-849D-14X-1,0 & 125.10 & 143.00 \\
\hline 138-846D-9H-2, 17 & 79.47 & 89.87 & I $38-853 \mathrm{~B}-2 \mathrm{H}-5,50$ & 10.80 & 10.75 & 138-849D-14X-1, 20 & 125.30 & 143.20 \\
\hline 138-846D-9H-2, 27 & 79.57 & 89.97 & $138-853 \mathrm{~B}-2 \mathrm{H}-5,55$ & 10.85 & 10.80 & 138-849D-14X-1, 40 & 125.50 & 143.40 \\
\hline $138-846 \mathrm{D}-9 \mathrm{H}-2,37$ & 79.67 & 90.07 & $138-853 \mathrm{~B}-2 \mathrm{H}-5.61$ & 10.91 & 10.86 & 138-849D-14X-1, 60 & 125.70 & 143.60 \\
\hline $138-846 \mathrm{D}-9 \mathrm{H}-2,47$ & 79.77 & 90.17 & $138-853 \mathrm{~B}-2 \mathrm{H}-5.65$ & 10.95 & 10.90 & $138-849 \mathrm{D}-14 \mathrm{X}-1.80$ & 125.90 & 143.80 \\
\hline $138-846 \mathrm{D}-9 \mathrm{H}-2,57$ & 79.87 & 90.27 & $138-853 \mathrm{~B}-2 \mathrm{H}-5,70$ & 11.00 & 10.95 & $138-849 \mathrm{D}-14 \mathrm{X}-1,100$ & 126.10 & 144.00 \\
\hline $138-847 \mathrm{C}-8 \mathrm{H}-1,40$ & 68.90 & 77.10 & I38-853B-2H-5, 75 & 11.05 & 11.00 & $138-849 D-14 X-1,120$ & 126.30 & 144.20 \\
\hline $138-847 \mathrm{C}-8 \mathrm{H}-1,50$ & 69.00 & 77.20 & $138-853 \mathrm{~B}-2 \mathrm{H}-5.78$ & 11.08 & 11.03 & $138-849 \mathrm{D}-14 \mathrm{X}-1,140$ & 126.50 & 144.40 \\
\hline $138-847 \mathrm{C}-8 \mathrm{H}-1,59$ & 69.09 & 77.29 & $138-853 \mathrm{~B}-2 \mathrm{H}-5.83$ & 11.13 & 11.08 & $138-849 \mathrm{D}-14 \mathrm{X}-2.10$ & 126.70 & 144.60 \\
\hline $138-847 \mathrm{C}-8 \mathrm{H}-1,70$ & 69.20 & 77.40 & 138-853B-2H-5, 88 & 11.18 & 11.13 & $138-849 \mathrm{D}-14 \mathrm{X}-2,30$ & 126.90 & 144.80 \\
\hline $138-847 \mathrm{C}-8 \mathrm{H}-1,80$ & 69.30 & 77.50 & $138-853 \mathrm{~B}-2 \mathrm{H}-5.93$ & 11.23 & 11.18 & $138-849 D-14 X-3,140$ & 129.50 & 147.40 \\
\hline $138-847 \mathrm{C}-8 \mathrm{H}-1.89$ & 69.39 & 77.59 & $138-853 \mathrm{~B}-2 \mathrm{H}-5,98$ & 11.28 & 11.23 & $138-849 \mathrm{D}-14 \mathrm{X}-4,10$ & 129.70 & 147.60 \\
\hline $138-847 \mathrm{C}-8 \mathrm{H}-1,100$ & 69.50 & 77.70 & $138-854 \mathrm{~B}-2 \mathrm{H}-4,14 \mathrm{I}$ & 14.31 & 14.61 & $138-849 \mathrm{D}-14 \mathrm{X}-4,30$ & 129.90 & 147.80 \\
\hline $138-847 \mathrm{C}-8 \mathrm{H}-1,110$ & 69.60 & 77.80 & $138-854 \mathrm{~B}-2 \mathrm{H}-5.2$ & 14.42 & 14.72 & $138-849 D-14 X-4,50$ & 130.10 & 148.00 \\
\hline $138-847 \mathrm{C}-8 \mathrm{H}-1,119$ & 69.69 & 77.89 & $138-854 \mathrm{~B}-2 \mathrm{H}-5.12$ & 14.52 & 14.82 & 138-849D-14X-4, 70 & 130.30 & 148.20 \\
\hline $138-848 \mathrm{C}-4 \mathrm{H}-1,0$ & 24.50 & 28.75 & $138-854 \mathrm{~B}-2 \mathrm{H}-5.22$ & 14.62 & 14.92 & $138-849 \mathrm{D}-14 \mathrm{X}-4.90$ & 130.50 & 148.40 \\
\hline $138-848 \mathrm{C}-4 \mathrm{H}-1,10$ & 24.60 & 28.85 & $138-854 \mathrm{~B}-2 \mathrm{H}-5,35$ & 14.75 & 15.05 & $138-849 \mathrm{D}-14 \mathrm{X}-4,110$ & 130.70 & 148.60 \\
\hline $138-848 \mathrm{C}-4 \mathrm{H}-1,15$ & 24.65 & 28.90 & & & & 138-849D-14-X-4, 130 & 130.90 & 148.80 \\
\hline $138-848 \mathrm{C}-4 \mathrm{H}-1,20$ & 24.70 & 28.95 & Time Slice 2: & & & $138-850 \mathrm{~B}-11 \mathrm{X}-2,11$ & 99.61 & 107.36 \\
\hline $138-848 \mathrm{C}-4 \mathrm{H}-\mathrm{I}, 30$ & 24.80 & 29.05 & $138-845 \mathrm{~B}-7 \mathrm{H}-3,110$ & 64.20 & 70.81 & $138-850 \mathrm{~B}-1 \mathrm{IX}-2,28$ & 99.78 & 107.53 \\
\hline $138-848 \mathrm{C}-4 \mathrm{H}-1,40$ & 24.90 & 29.15 & $138-845 \mathrm{~B}-7 \mathrm{H}-3,120$ & 64.30 & 70.91 & $138-850 \mathrm{~B}-11 \mathrm{X}-2,38$ & 99.88 & 107.63 \\
\hline $138-848 \mathrm{C}-4 \mathrm{H}-1,50$ & 25.00 & 29.25 & $138-845 \mathrm{~B}-7 \mathrm{H}-3,129$ & 64.39 & 71.00 & $138-850 \mathrm{~B}-11 \mathrm{X}-2,47$ & 99.97 & 107.72 \\
\hline $138-848 \mathrm{C}-4 \mathrm{H}-1,60$ & 25.10 & 29.35 & $138-845 \mathrm{~B}-7 \mathrm{H}-3,140$ & 64.50 & 71.11 & $138-850 \mathrm{~B}-11 \mathrm{X}-2.57$ & 100.07 & 107.82 \\
\hline $138-848 \mathrm{C}-4 \mathrm{H}-1,70$ & 25.20 & 29.45 & $138-845 \mathrm{~B}-7 \mathrm{H}-4,110$ & 65.70 & 72.31 & $138-850 \mathrm{~B}-11 \mathrm{X}-5.32$ & 104.32 & 112.07 \\
\hline $138-848 \mathrm{C}-4 \mathrm{H}-1,80$ & 25.30 & 29.55 & $138-845 \mathrm{~B}-7 \mathrm{H}-4,120$ & 65.80 & 72.41 & $138-850 \mathrm{~B}-11 \mathrm{X}-5,42$ & 104.42 & 112.17 \\
\hline $138-848 \mathrm{C}-4 \mathrm{H}-1,90$ & 25.40 & 29.65 & $138-845 \mathrm{~B}-7 \mathrm{H}-4.130$ & 65.90 & 72.51 & $138-850 \mathrm{~B}-11 \mathrm{X}-5,52$ & 104.52 & 112.27 \\
\hline $138-848 \mathrm{C}-4 \mathrm{H}-1.95$ & 25.45 & 29.70 & $138-845$ B-7H-4, 139 & 65.99 & 72.60 & $138-850 B-11 X-5,62$ & 104.62 & 112.37 \\
\hline $138-848 \mathrm{C}-4 \mathrm{H}-1.100$ & 25.50 & 29.75 & $138-846 \mathrm{D}-18 \mathrm{H}-1,14$ & 163.44 & 185.54 & $138-850 \mathrm{~B}-11 \mathrm{X}-5,72$ & 104.72 & 112.47 \\
\hline $138-848 \mathrm{C}-4 \mathrm{H}-1,105$ & 25.55 & 29.80 & $138-846 \mathrm{D}-18 \mathrm{H}-1.24$ & 163.54 & 185.64 & $138-850 \mathrm{~B}-11 \mathrm{X}-5,87$ & 104.87 & 112.62 \\
\hline $138-848 \mathrm{C}-4 \mathrm{H}-1,110$ & 25.60 & 29.85 & 138-846D-18H-1. 34 & 163.64 & 185.74 & 138-851E-9H-3, 7 & 79.87 & 90.92 \\
\hline $138-848 \mathrm{C}-4 \mathrm{H}-1,115$ & 25.65 & 29.90 & $138-846 \mathrm{C}-18 \mathrm{H}-2,130$ & 166.80 & 190.00 & 138-851E-9H-3, 97 & 79.97 & 91.02 \\
\hline $138-848 \mathrm{C}-4 \mathrm{H}-1,120$ & 25.70 & 29.95 & $138-846 \mathrm{C}-18 \mathrm{H}-2,140$ & 166.90 & 190.10 & I38-851E-9H-3, 107 & 80.07 & 91.12 \\
\hline $138-848 \mathrm{C}-4 \mathrm{H}-1,125$ & 25.75 & 30.00 & $138-846 \mathrm{C}-18 \mathrm{H}-3,0$ & 167.00 & 190.20 & $138-851 \mathrm{E}-9 \mathrm{H}-3,117$ & 80.17 & 91.22 \\
\hline $138-848 \mathrm{C}-4 \mathrm{H}-1,130$ & 25.80 & 30.05 & $138-846 \mathrm{C}-18 \mathrm{H}-3,10$ & 167.10 & 190.30 & 138-851E-9H-3. 127 & 80.27 & 91.32 \\
\hline $138-848 \mathrm{C}-4 \mathrm{H}-1,135$ & 25.85 & 30.10 & $138-846 \mathrm{C}-18 \mathrm{H}-3,20$ & 167.20 & 190.40 & 138-851E-9H-3, 137 & 80.37 & 91.42 \\
\hline $138-848 \mathrm{C}-4 \mathrm{H}-1.140$ & 25.90 & 30.15 & $138-847 \mathrm{C}-16 \mathrm{X}-1,82$ & 145.62 & 165.02 & |38-85IE-9H-5, 77 & 82.77 & 93.82 \\
\hline 138-849D-6H-5, 85 & 58.35 & 67.60 & $138-847 \mathrm{C}-16 \mathrm{X}-1,102$ & 145.82 & 165.22 & 138-85IE-9H-5, 87 & 82.87 & 93.92 \\
\hline 138-849D-6H-5, 95 & 58.45 & 67.70 & $138-847 \mathrm{C}-16 \mathrm{X}-1.122$ & 146.02 & 165.42 & 138-851E-9H-5, 97 & 82.97 & 94.02 \\
\hline 138-849D-6H-5, 105 & 58.55 & 67.80 & $138-847 \mathrm{C}-16 \mathrm{X}-1,142$ & 146.22 & 165.62 & 138-851E-9H-5, 107 & 83.07 & 94.12 \\
\hline 138-849D-6H-5, 115 & 58.65 & 67.90 & $138-847 \mathrm{C}-16 \mathrm{X}-2,3$ & 146.33 & 165.73 & 138-851E-9H-5, 117 & 83.17 & 94.22 \\
\hline 138-849D-6H-5, 125 & 58.75 & 68.00 & $138-847 \mathrm{C}-16 \mathrm{X}-2,23$ & 146.53 & 165.93 & $138-851 \mathrm{E}-9 \mathrm{H}-5,127$ & 83.27 & 94.32 \\
\hline 138-849D-6H-5, 135 & 58.85 & 68.10 & $138-847 \mathrm{C}-16 \mathrm{X}-2,43$ & 146.73 & 166.13 & 138-85IE-9H-5, 137 & 83.37 & 94.42 \\
\hline 138-849D-6H-5, 145 & 58.95 & 68.20 & $138-847 C-16 X-2,63$ & 146.93 & 166.33 & I38-852C-6H-4, 25 & 48.75 & 53.75 \\
\hline 138-849D-6H-6, 5 & 59.05 & 68.30 & $138-847 \mathrm{C}-16 \mathrm{X}-2,83$ & 147.13 & 166.53 & $138-852 \mathrm{C}-6 \mathrm{H}-4,30$ & 48.80 & 53.80 \\
\hline $138-849 \mathrm{D}-6 \mathrm{H}-6,15$ & 59.15 & 68.40 & $138-847 \mathrm{C}-16 \mathrm{X}-2,103$ & 147.33 & 166.73 & $138-852 \mathrm{C}-6 \mathrm{H}-4,35$ & 48.85 & 53.85 \\
\hline $138-849 \mathrm{D}-6 \mathrm{H}-6,25$ & 59.25 & 68.50 & $138-847 \mathrm{~B}-16 \mathrm{X}-3,119$ & 143.69 & 160.14 & $138-852 \mathrm{C}-6 \mathrm{H}-4,40$ & 48.90 & 53.90 \\
\hline $138-849 \mathrm{D}-6 \mathrm{H}-6.45$ & 59.45 & 68.70 & $138-847 \mathrm{~B}-16 \mathrm{X}-3,139$ & 143.89 & 160.34 & $138-852 \mathrm{C}-6 \mathrm{H}-4,45$ & 48.95 & 53.95 \\
\hline $138-849 \mathrm{D}-6 \mathrm{H}-6,45$ & 59.45 & 68.70 & $138-847 \mathrm{~B}-16 \mathrm{X}-4.3$ & 144.06 & 160.51 & $138-852 \mathrm{C}-6 \mathrm{H}-4,50$ & 49.00 & 54.00 \\
\hline $138-849 \mathrm{D}-6 \mathrm{H}-6,55$ & 59.55 & 68.80 & $138-847 \mathrm{~B}-16 \mathrm{X}-4,5$ & 144.35 & 160.80 & $138-852 \mathrm{C}-6 \mathrm{H}-4,55$ & 49.05 & 54.05 \\
\hline $138-850 \mathrm{~B}-5 \mathrm{H}-2,90$ & 43.40 & 47.80 & $138-847 \mathrm{~B}-16 \mathrm{X}-4,54$ & 144.54 & 160.99 & $138-852 \mathrm{C}-6 \mathrm{H}-4,60$ & 49.10 & 54.10 \\
\hline $138-850 \mathrm{~B}-5 \mathrm{H}-2,100$ & 43.50 & 47.90 & $138-847 \mathrm{~B}-16 \mathrm{X}-4,70$ & 144.70 & 161.15 & $138-852 \mathrm{C}-6 \mathrm{H}-5,25$ & 50.25 & 55.25 \\
\hline $138-850 \mathrm{~B}-5 \mathrm{H}-2,111$ & 43.61 & 48.01 & $138-847 \mathrm{~B}-16 \mathrm{X}-4,91$ & 144.91 & 161.36 & $138-852 \mathrm{C}-6 \mathrm{H}-5,30$ & 50.30 & 55.30 \\
\hline $138-850 \mathrm{~B}-5 \mathrm{H}-2,121$ & 43.71 & 48.11 & $138-848 \mathrm{C}-5 \mathrm{H}-6,0$ & 41.50 & 46.05 & $138-852 \mathrm{C}-6 \mathrm{H}-5,35$ & 50.35 & 55.35 \\
\hline $138-850 \mathrm{~B}-5 \mathrm{H}-2,130$ & 43.80 & 48.20 & $138-848 \mathrm{C}-5 \mathrm{H}-6.5$ & 41.55 & 46.10 & $138-852 \mathrm{C}-6 \mathrm{H}-5,40$ & 50.40 & 55.40 \\
\hline $138-850 \mathrm{~B}-5 \mathrm{H}-2,140$ & 43.90 & 48.30 & $138-848 \mathrm{C}-5 \mathrm{H}-6,10$ & 41.60 & 46.15 & $138-852 \mathrm{C}-6 \mathrm{H}-5,45$ & 50.45 & 55.45 \\
\hline $138-850 \mathrm{~B}-5 \mathrm{H}-3, \mathrm{I}$ & 44.01 & 48.41 & $138-848 \mathrm{C}-5 \mathrm{H}-6,15$ & 41.65 & 46.20 & $138-852 \mathrm{C}-6 \mathrm{H}-5,50$ & 50.50 & 55.50 \\
\hline $138-85 \mid \mathrm{C}-4 \mathrm{H}-5,53$ & 37.53 & 44.38 & $138-848 \mathrm{C}-5 \mathrm{H}-6.20$ & 41.70 & 46.25 & $138-852 \mathrm{C}-6 \mathrm{H}-5,55$ & 50.55 & 55.55 \\
\hline $138-851 \mathrm{IC}-4 \mathrm{H}-5,58$ & 37.58 & 44.43 & $138-848 \mathrm{C}-5 \mathrm{H}-6,30$ & 41.80 & 46.35 & $138-852 \mathrm{C}-6 \mathrm{H}-5,60$ & 50.60 & 55.60 \\
\hline
\end{tabular}

Mix (unpubl. data). Unlike previous efforts to develop transfer functions with radiolarian data (Sachs, 1973; Moore et al., 1980), these data were first log-transformed. This transformation has the property of reducing the influence of a single, very abundant species on defining individual factors. In the original transfer function approach of Imbrie and Kipp (1971), a percent range transformation was used. The percent range transformation has a number of disadvantages: (1) if all species abundance data are scaled to range from 0 (equal to the minimum occurrence) to 100 (the maximum values), the amount of noise in the data, especially for rare forms, is magnified; and (2) the percent range transformation introduces unnecessary zeros into the data set. The $Q$-mode factor analysis used by Imbrie and Kipp (1971) is an approach that uses intervariable ratios as the method of distinguishing sample differences, and the transformation of data to zeros fundamentally changes these ratios. A $\log$ transform was used to avoid these problems. The transformation does not increase the variance of any species weighting and thus does not seem to increase data noise. The transformation assigns the value of zero only to variables 
A
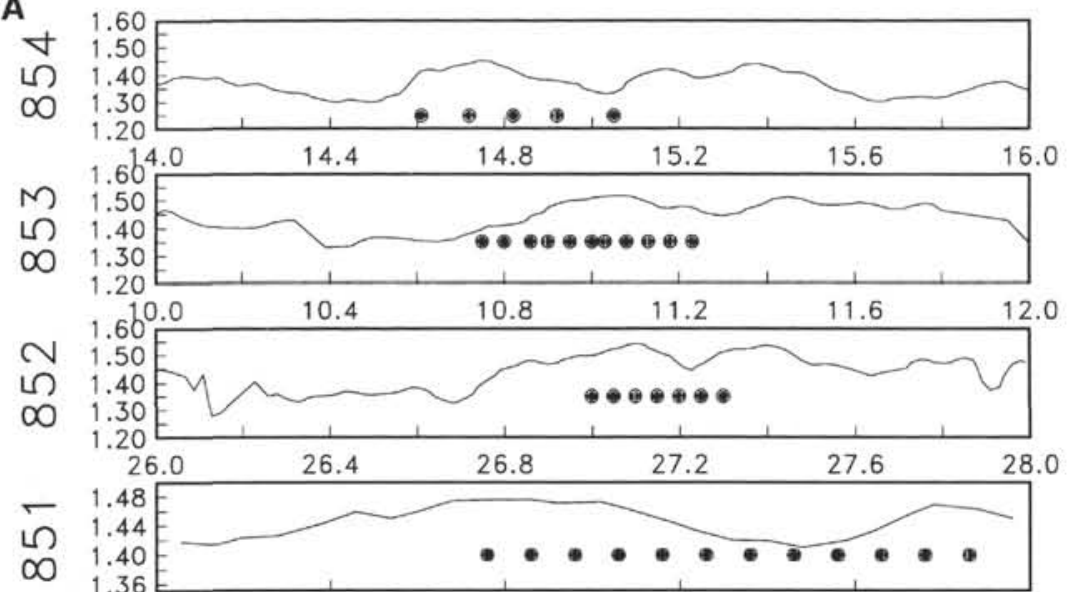

$\infty \quad 1.36$

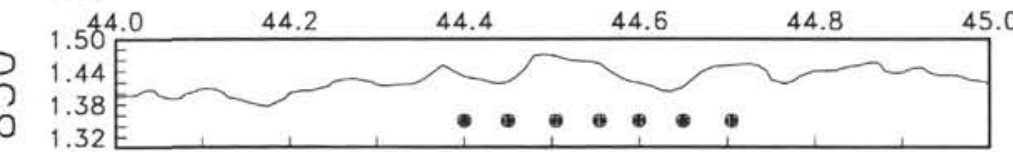

$\infty \quad 1.38$

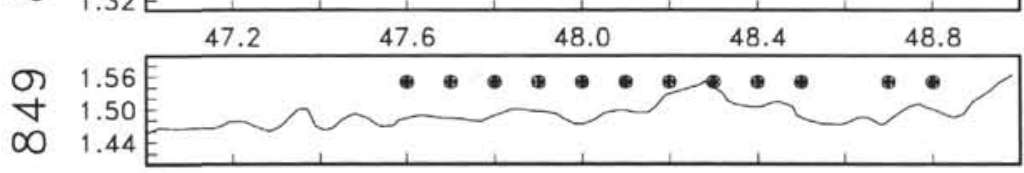

$\infty 1.44$
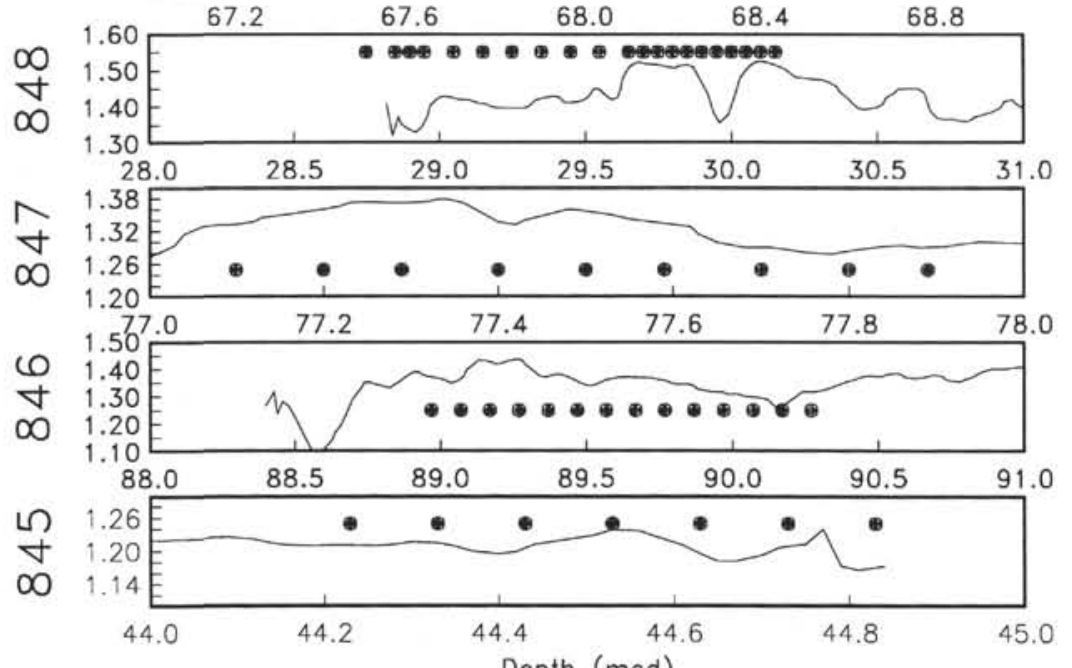

B

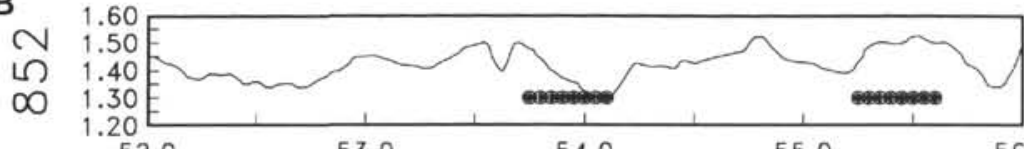

$$
\infty
$$

52.0

53.0

54.0 56.0

एक

$\infty 1$

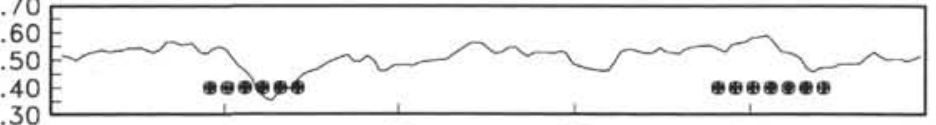

$1.70^{90}$

91

92

93

94 95

$\infty$

$\infty 1$.

=

1.40
1.30 $\begin{array}{lllllll}107 & 108 & 109 & 110 & 111 & 112 & 113\end{array}$

or $1.60 \mathrm{H}^{2}$

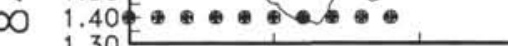

$143 \quad 144 \quad 145 \quad 146 \quad 147$

148

$\begin{array}{ll}\infty & 1.60 \\ \forall & 1.50\end{array}=\bullet \bullet \bullet \bullet \bullet \bullet \bullet \bullet \bullet \bullet \bullet \bullet \bullet \bullet \bullet \bullet$

$\infty \quad 1.30=$

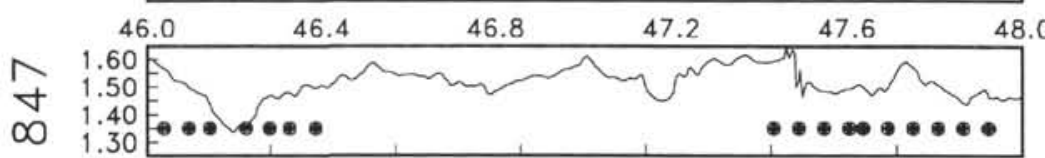

$\infty 1.30$
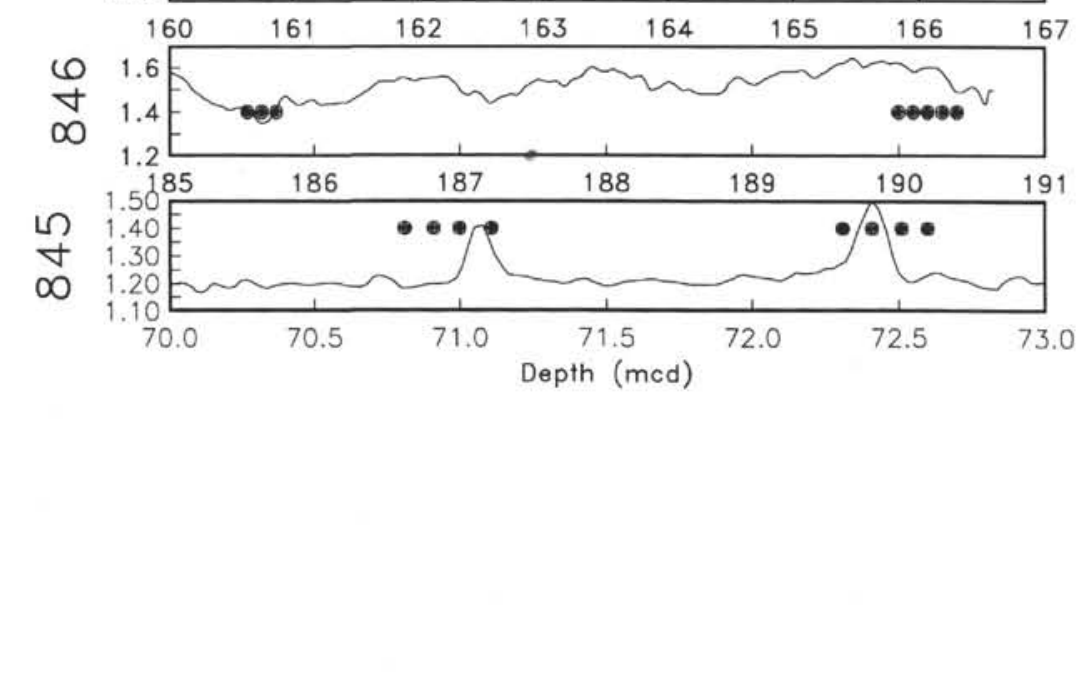

Figure 1. GRAPE density records for time slice studies. A. GRAPE density records, plotted vs. meters composite depth, for Sites 845 through 854 in the interval from approximately 2.3 to 2.5 Ma. Solid dots show where samples were taken for radiolarian analysis. B. GRAPE density records, plotted vs. meters composite depth, for Sites 845 through 852 for the interval from 4.7 to 5.0 Ma. 
Table 2. Radiolarian species used in this study.

\begin{tabular}{|c|c|c|}
\hline $\begin{array}{l}\text { Laboratory } \\
\text { code }\end{array}$ & Species name & Reference \\
\hline $\mathrm{Si}^{+}$ & Spongurus sp. & 3 p. 33 , pl. I, fig. 2 \\
\hline $\mathrm{S}_{\mathrm{A}}^{+}$ & Spongurus elliptica & p. 563 , pl. 8 , fig. 2 \\
\hline S3 & $\begin{array}{l}\text { Actinomma arcadophorum and } A \text {. } \\
\text { medianum }\end{array}$ & ${ }^{1}$ p. S31, pl. 3, figs. 5-6 \\
\hline S4** & Actinomma leptodermum & p. S35, pl. 3, fig. 7 \\
\hline $\mathrm{S} 7^{+}$ & Echinomma cf. leptodermum & p. 258 , pl. 3 , fig. 6 \\
\hline $\mathrm{S} 8^{+}$ & Prunopyle antarctica & p. S127, pl. 16, fig. 4 \\
\hline S9 & Amphirhopalum ypsilon & p. S75, pl.10, fig. 1 \\
\hline $\mathrm{S}_{1} 0^{+}$ & Echinomma delicatum & p. 333 , pl. 1, fig. 5 \\
\hline SII & Collosphaera tuberosa & p. S1, pl. I, fig. I \\
\hline $\mathrm{S} 12^{+}$ & Euchitonia furcata and $E$. elegans & $\begin{array}{l}\text { p. S85, pl. } 11 \text {, figs. } 2 \mathrm{~A}-2 \mathrm{~B} \\
\text { p. } 83 \text {, pl, } 11 \text {, figs. } 1 \mathrm{~A}-1 \mathrm{~B}\end{array}$ \\
\hline $\mathrm{S}_{3} 3^{+}$ & Polysolenia spinosa & p. SI9, pl. 2 , fig. 5 \\
\hline $\mathrm{S}_{1} 4^{+}$ & Heliodiscus astericus & p. S73, pl. 9, figs. 1-2 \\
\hline S15 & Actinomma antarcticum & p. S25, pl. 3., figs. I-3 \\
\hline $\mathrm{S} 17^{+}$ & Hexacontium enthacanthum & p. S45, pl. 5, figs. IA-1B \\
\hline S18 $8^{+}$ & Hymenastrium euclidis & p. S91, pl. 12, fig. 3 \\
\hline S19 & Larcospira quadrangula & p. S133, pl. 17, fig. 2 \\
\hline S21 & Cenosphaera coronata & p. S39, pl. 4, fig. I \\
\hline $\mathrm{S} 23^{+}$ & Didymocyrtis tetrathalamus & p. S49, pl. 6, fig. I \\
\hline S $24^{+}$ & Lithelius minor & p. S135, pl. 17 , figs. $3-4$ \\
\hline S29* & Larcopyle butschlii & p. S131, pl. 17, fig. I \\
\hline $\mathrm{S} 30^{+}$ & Stylochlamydium asteriscus & p. 5113, pl. 14 , fig. 5 \\
\hline S32 & Polysolenia arktios & p. S11, pl. 2, fig. I \\
\hline S33 & Polysolenia lappacea & p. S15, pl. 2, figs. $3 \mathrm{~A}-3 \mathrm{~B}$ \\
\hline $\mathrm{S} 34^{* *}$ & Polysolenia murrayana & p. S17, pl. 2 , fig. 4 \\
\hline S36 & Dictyocoryne truncatum & p. S89, pl. 12, fig. $2 \mathrm{~A}$ \\
\hline $\mathrm{S} 36 \mathrm{~A}^{+}$ & Dictyocoryne profunda & p. S87, pl. 12, fig. 1 \\
\hline $\mathrm{S} 36 \mathrm{C}^{+}$ & Euchitonia triangulum & p. 10 , pl. 6 , fig. 8 \\
\hline $\mathrm{S} 40^{+}$ & Spongaster tetras & p. S93, pl. 13, fig. 1 \\
\hline S41** & Spongurus pylomaticus & p. 261 , pl. 4 , figs. $8-9$ \\
\hline \$42 & Spongocore puella & p. S69, pl. 8, fig. 5 \\
\hline $\mathrm{S}^{3} 3^{+}$ & Spongopyle osculosa & p. 334 , pl. V, fig. 18 \\
\hline S44 & Spongotrochus glacialis & p. S117, pl. 15, fig. 2 \\
\hline $\mathrm{S} 47^{+}$ & Stylodictya validispina & p. Si03, pl. 13 , fig. 5 \\
\hline $\mathrm{S} 48^{+}$ & Porodiscus(?) sp. B & p. S109, pl. 14 , figs. $3-4$ \\
\hline S50 & Axoprunium stauraxonium & p. S57, pl. 7, figs. 2-3 \\
\hline S $51^{* *}$ & Stylatractus spp. & p. S55, pl. 7, fig. I \\
\hline S52 & Styptosphaera spumacea & p. S71, pl. 8, fig. 6 \\
\hline S53 & Hexapyle spp. & p. S121,pl. 16, fig. 1 \\
\hline $\mathrm{S} 54^{+}$ & $\begin{array}{l}\text { Tetrapyle octacantha and Octopyle } \\
\text { stenozona }\end{array}$ & p. S123-S125, pl. 16 , figs. $2-3$ \\
\hline CIN & Buccinospheara invaginata & 9 pl. I, figs. 5-6 \\
\hline SLB & Lithosphaera bacca & 16 pl. 9 , fig. 13 \\
\hline $\mathrm{NI}^{+}$ & Liriospyris reticulata & p. N13, pl. 19, fig. 4 \\
\hline $\mathrm{NIC}$ & Zygocircus sp. & pl. 20 , figs. 2,8 , and 9 \\
\hline $\mathrm{N} 2 *$ & Anthocyrtidium ophirense & p. N67, pl. 25, fig. I \\
\hline N3 & Anthocyrtidium zanguebaricum & p. N69, pl. 25, fig. 2 \\
\hline $\mathrm{N} 4$ & Carpocanistrum spp. & p. N23, pl. 21 , fig. I \\
\hline N5* & Lamprocyrtis nigriniae & p. N81, pl. 24 , fig. 7 \\
\hline $\mathrm{N}^{+}$ & Pterocorys minithorax & p. N87, pl. 25 , fig. 10 \\
\hline N8 & Carpocanistrum papillosum & ${ }^{1}$ p. N27, pl. 21 , fig. 3 \\
\hline GN8 & Ceratospyris polygona & p. NI5, pl. 19, fig. 5 \\
\hline $\mathrm{N}^{+}$ & Giraffospyris angulata & p. N11, pl. 19, figs. 2A-2D \\
\hline NIO & Eucyrtidium acuminatum & 5 p. 275 ., pl. 9. fig. 5 \\
\hline NII & Eucyrtidium hexagonatum & p, N63, pl. 24 , fig. 4 \\
\hline $\mathrm{N}_{4} 4^{+}$ & Tholospyris scaphipes & p. N19, pl. 20, fig. 2 \\
\hline GN14 & Desmospyris anthocyrtoides & ${ }^{2}$ p. 332 , pl. 23 , figs. $6-8$ \\
\hline
\end{tabular}

\begin{tabular}{|c|c|c|}
\hline $\begin{array}{l}\text { Laboratory } \\
\text { code }\end{array}$ & Species name & Reference \\
\hline N15* & Lamprocyclas junonis & p. 337, pl. VII, fig. 10 \\
\hline N16 & Lamprocyclas maritalis var. & $\begin{array}{l}\text { p. N77, pl. } 25 \text {, fig. } 5 ; \\
\text { p. N79, pl. } 25 \text {, fig. } 6\end{array}$ \\
\hline N17 & Lamprocyclas maritalis maritalis & p. N75, pl. 25, fig. 4 \\
\hline $\mathrm{N} 18^{+}$ & Botryostrobus auritus/australis Group & p. N101, pl. 27 , fig. 2 \\
\hline N19 & Botryocyrtis scutum & p. N105, pl. 28 , figs. $1 \mathrm{~A}-1 \mathrm{~B}$ \\
\hline $\mathrm{N} 24^{*}$ & Pterocanium sp. & p. N49, pl. 23, fig. 6 \\
\hline $\mathrm{N} 27^{* * *}$ & Plerocanium praetextum and P. eucolpum & p. N43, pl. 23, fig. 3 \\
\hline N28 & Pterocanium trilobum & p. N45, pl. 23 , fig. 4 \\
\hline $\mathrm{N} 29^{+}$ & Dictyophimus hirundo & p. N35, pl. 22, figs. 2-4 \\
\hline $\mathrm{N} 32^{*}$ & Phormostichoartus corbula & p. N103, pl. 27 , fig. 3 \\
\hline $\mathrm{N}^{3} 3^{+}$ & Botryostrobus aquilonaris & p. N99, pl. 27, fig. 1 \\
\hline N35* & Theocalyptra davisiana davisiana & ${ }_{7}^{7} \mathrm{pl} 1 .$, figs. $1-5$ \\
\hline $\mathrm{N}_{35 \mathrm{~A}^{+}}$ & Theocalyptra davisiana cormutoides & ${ }^{7} \mathrm{pl}$ 1., figs. 6-10 \\
\hline N36** & Theocalyptra bicornis var. & pl. 15, figs. 4-5 \\
\hline $\mathrm{N} 38^{+}$ & Theocalyptra bicornis & pl. 15 , fig. 6 \\
\hline N39 & Pterocorys hertwigii & p. N85, pl. 25 , fig. 9 \\
\hline $\mathrm{N} 40^{+}$ & Pterocorys clausus & 6 pl. 1 , figs. 6,7 , and 10 \\
\hline $\mathrm{N} 42^{*}$ & Theocorythium trachelium trachelium & p. N93-N95, pl. 26, figs. $2-3$ \\
\hline $\mathrm{N} 43^{*}$ & Ceratospyris borealis & p. N9, pl. 19, figs. IA-1D \\
\hline EI & Lamprocyrtis neoheteroporus & 11 p. 639 , pl. 5, figs. $17-18$ \\
\hline E2 & Lamprocyrtis heteroporus & 11 p. 639, pl. 5, figs. 19-21 \\
\hline E3 & Theocorythium vetulum & p. NI69, pl. 30 , figs. $4 \mathrm{~A}-4 \mathrm{~B}$ \\
\hline E4 & Didymocyrtis avita & ${ }^{12}$ p. 808 , pl. 84, fig. 7 \\
\hline E5 & Didymocyrtis penuitima & ${ }^{8}$ p. S57, pl. 7, figs. 3A-3C \\
\hline E6 & Stylatractus universus & p. S29, pl. 4 , fig. 3 \\
\hline E7 & Pterocanium prismatium & p. N125, pl. 25 , fig. 2 \\
\hline E8 & Stichocorys peregrina & p. NI33, pl. 25 , fig. 6 \\
\hline E9 & Spongaster pentas & p. S65, pl. 9, fig. 2 \\
\hline E10 & Phormostichoartus fistula & p. NI83, pl. 31, figs. $6 \mathrm{~A}-6 \mathrm{C}$ \\
\hline E11 & Phormostichoartus doliolum & p. N181, pl. 31, figs. 5A-5B \\
\hline E12 & Botryostrobus bramlettei & p. N137, pl. 31, figs. $2 \mathrm{~A}-2 \mathrm{C}$ \\
\hline E13 & Lychnodictyum audax & p. N123, pl. 25, fig. I \\
\hline E14 & Phormostichoartus marylandicus & ${ }^{8}$ p. N185, pl. 31, figs. 7A-7E \\
\hline E15 & Solenosphaera omnitubus omnitubus & p. S7, pl. 1, fig. 4 \\
\hline E16 & Stichoconys delmontensus & ${ }_{\text {p. N129, pl. } 25, \text { fig. } 4}$ \\
\hline E17 & Stichoconys sp. & 8 \\
\hline $\mathrm{N} 46 \%$ & Antartissa denticulata & 'p. NI, pl. 18, figs. IA-1B \\
\hline
\end{tabular}

*Species used only for the analysis of Time Slice 1.

**Species used only for the analysis of Time Slice 2.

+Species used for both time slices.

${ }^{1}$ Nigrini and Moore (1979).

'Benson (1966).

${ }^{3}$ Molina-Cruz (1977).

${ }^{4}$ Moore (1974).

${ }^{5}$ Robertson (1975).

${ }^{6}$ Caulet and Nigrini (1988).

${ }^{7}$ Morley (1980)

${ }^{8}$ Nigrini and Lombari (1984).

${ }^{9}$ Knoll and Johnson (1975).

${ }^{10}$ Takahashi and Honjo (1981).

"Kling (1973).

${ }^{12}$ Riedel (1953).

that already have zero values (the $\log$ transform used is $y=\log [x+1]$, where $y$ is the transformed data and $x$ is the proportion of a given species in a sample). In the development of a new paleotemperature transfer function for late Pleistocene samples, we also found that the statistical properties (the standard error of estimates) of the transfer function using a log-transform were improved relative to untransformed data (N.G. Pisias and A.C. Mix, unpubl. data).

The seven factors, or "assemblages," account for more than $90 \%$ of Pacific wide data set (Figs. 3-5). We show the distribution of three of the seven factors identified in the surface data set. These three factors were found to be the most informative with respect to the Leg 138 time slice study. Together, these three factors account for about $84 \%$ of the total surface data set; one should note, however, that selection of a factor based purely on how much of the surface data set it accounted for would be inappropriate. In a $Q$-mode factor analysis as used in this study, each sample is represented by a unit-length vector and the total information contained in the data set is defined as the number of samples (which is the sum of the length of all sample vectors). Factor analysis describes all sample vectors as a sum of new

assemblages or factors. The vector length of this new description of each sample is called the communality. If a sample is perfectly described by the factor analysis model, then the communality is equal to 1 . The sum of all the communalities gives the estimate of the amount of information contained in the factor analysis and, when expressed as a percentage of the original total (the number of samples), yields the percentage of the data set explained by the factor analysis. When interpreting the amount of information contained in any one factor, care must be taken to recognize the nonrandom distribution of the original data set. For example, a large fraction of the radiolarian surface samples are from the tropical and subtropical parts of the Pacific, with only a few samples from the true Antarctic. Thus, one can expect that a factor describing the Antarctic assemblage would not account for as large a fraction of the data set as a factor describing the tropical or tropical assemblage. Clearly, to describe the variability in the Antarctic, it is more appropriate to use an assemblage important to that region.

The most important assemblage found in the surface data set (i.e., the tropical factor; Fig. 3) accounts for $57 \%$ of the data set. The 

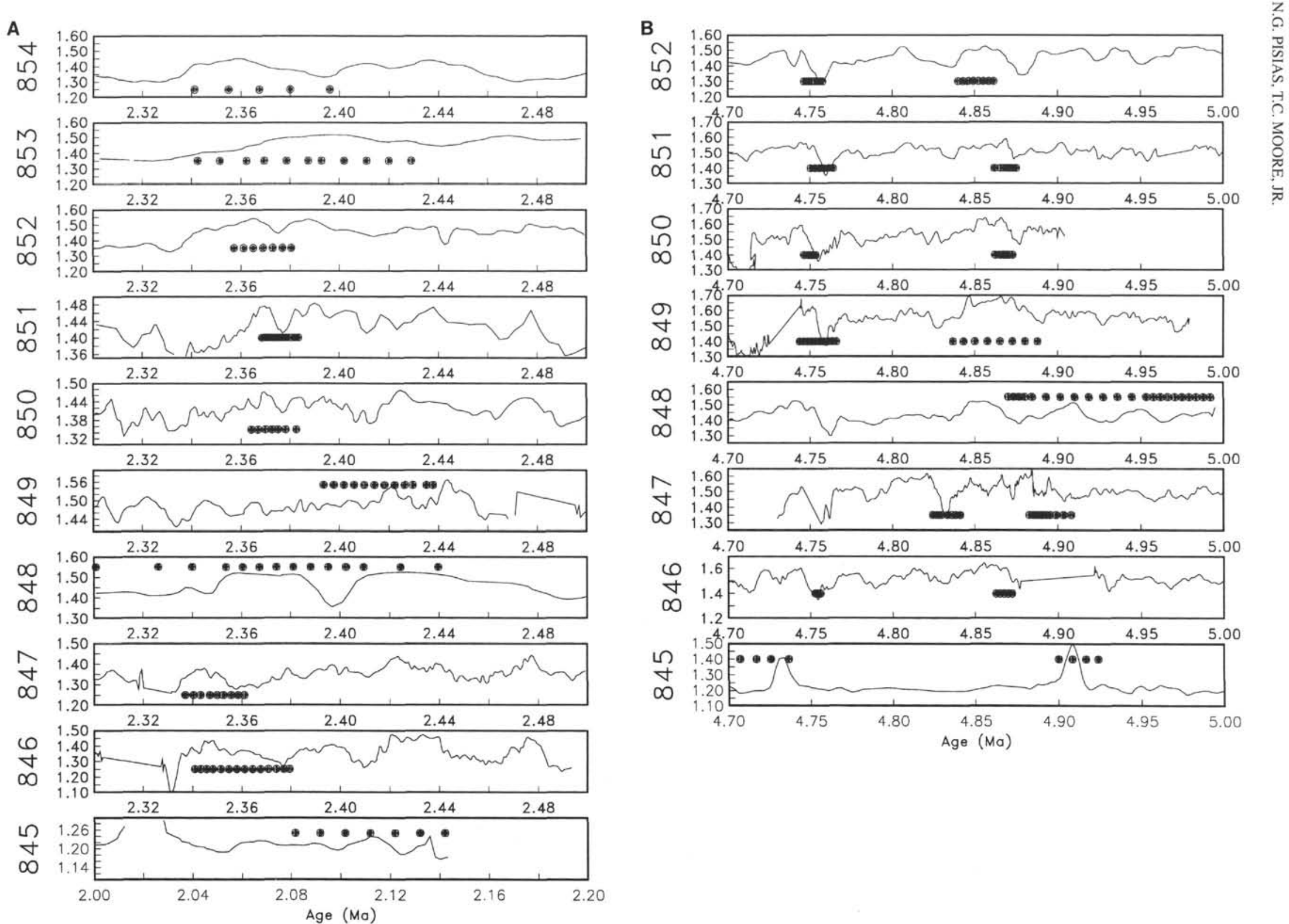

Figure 2. Same as Figure 1, but data have been plotted vs. age. 


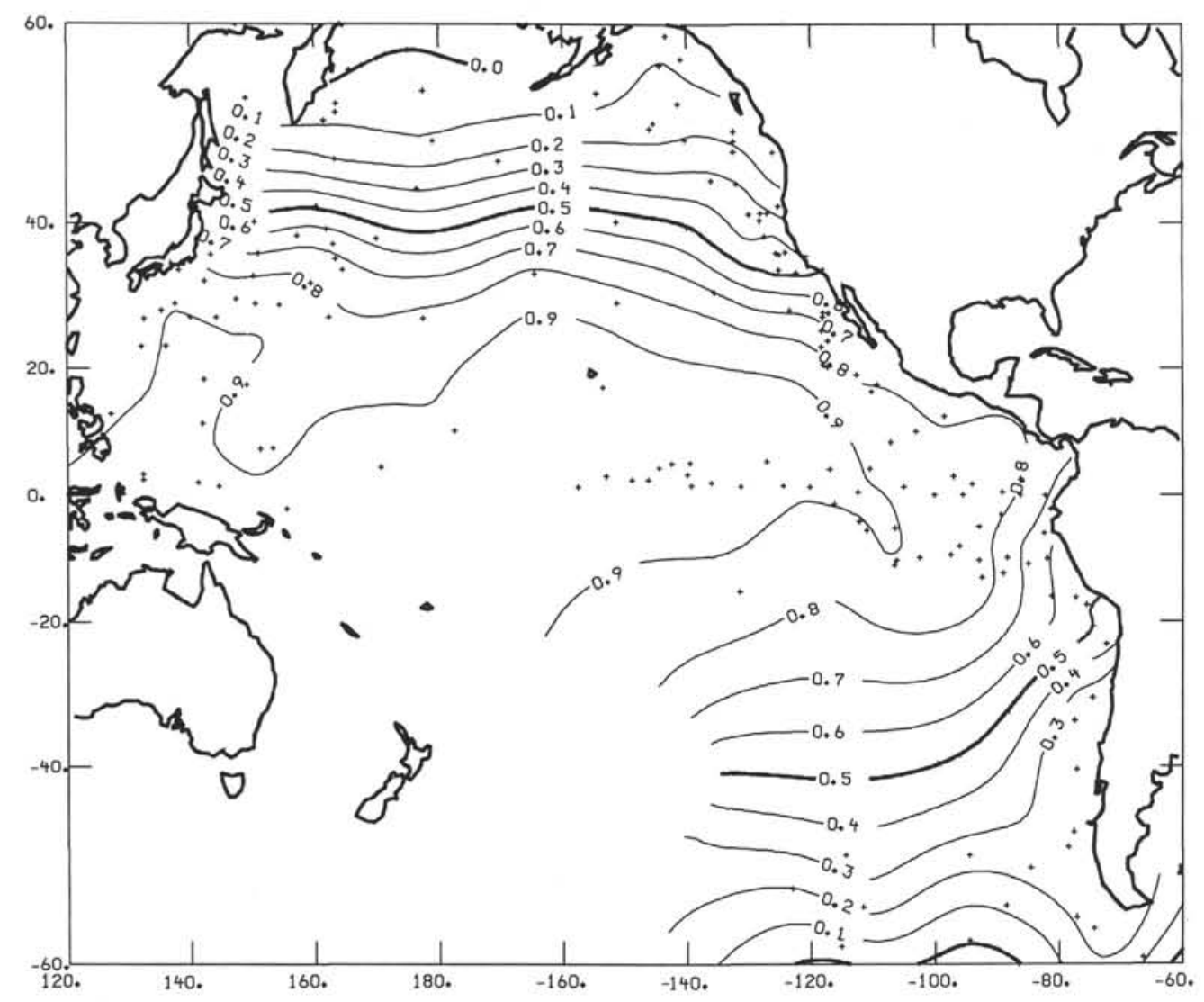

Figure 3. Distribution map of the factor loadings of the first (tropical) radiolarian assemblage extracted from analysis of surface sediments from the Pacific Ocean.

important components of this assemblage are species group Tetrapyle octacantha and Octopyle stenozoa, and the species Stylochlamydium asteriscus and Didymocyrtis tetrathalamus. This assemblage is most important in samples from the subtropical regions of the Pacific and is equivalent to the "tropical" assemblage identified by Moore (1978).

The second assemblage (the "transitional" assemblage) accounts for about $23 \%$ of the total data information and is found in the oceanic transition regions of both the North and South Pacific (Fig. 4). This assemblage is dominated by Lithelius minor and Botryostrobus aquilonaris, with species Larcopyle butschlii, Echinomma delicatum, Stylodictya validispina, Prunopyle antarctica, and Pterocorys clausus also being important. This assemblage is very similar to the "transition" assemblage of Moore (1978). The dominant species of this assemblage in both studies are the same.

The third assemblage relevant to this study is found along the eastern boundary of the Pacific and within the Northwest Pacific (Fig. 5). In the surface sediment data set, this assemblage accounts for $4 \%$ of the total data set. The most important species in this assemblage are Spongurus sp., Lamprocyrtis nigriniae, Pterocorys clausus, Pterocorys minithorax, and Theocalyptra davisiana davisiana. The biregional distribution of this assemblage reflects the abundance of T. davisiana davisiana in the region of the Sea of Okhotsk and the importance of this species in the eastern boundary current regions of the Pacific.

Because all of the species used in the surface sediment factor analysis are extant during our Time Slice 1, we use this factor model to simplify the Time Slice 1 data set. The factor score matrix from the surface sediment analysis (Table 3 ) was used to predict the relative importance of these modern-day assemblages in the samples from Time Slice 1 . As with the original factor analysis of the surface data, the Time Slice 1 data matrix first was normalized to consist of unit length vectors. The product of this matrix and the factor score matrix is the estimated factor loading matrix for the time slice samples (Table 4).

Because many of the species used in radiolarian studies of recent sediments are not present during Time Slice 2, we use, for this preliminary analysis, the species assemblages identified by Hagelberg and Pisias (1990). Hagelberg and Pisias (1990) analyzed radiolarian assemblages from the Pliocene section of Deep Sea Drilling Project (DSDP) Site 572. To extract paleoceanographic information, a paleotemperature transfer function was generated specifically for the Pliocene data set from Site 572. This factor analysis is based on 35 species, and six assemblages accounted for $91 \%$ of the information contained in this reduced data set. In this analysis the data was not log-transformed. The 35 species were selected based on comparisons between their relative abundances found in the Pliocene sediments of Sites 572 and 573 and in surface sediments from the Pacific.

For the analysis presented here, we concentrate on the pattern of change for the tropical, western Pacific and the Eastern Boundary Current (EBC) assemblage (Arctic/Peru Upwelling assemblage of Hagelberg and Pisias, 1990). As with the assemblages used to analyze Time Slice 1, the tropical assemblage identified in this more limited factor analysis is dominated by $T$. octacantha and $O$. stenozona. An important difference between this limited factor analysis and the analysis used for the younger time slice is the identification of a western Pacific assemblage dominated by S. asteriscus, which for the most part is included in the tropical assemblage shown in Figure 3. The EBC assemblage identified by Hagelberg and Pisias (1990) is dominated by $P$. minithorax, Spongurus sp., Polysolenia murrayana, and Echinomma delicatum. The first two species in this assemblage are also important in the EBC assemblage shown in Figure 5. However, two species important in defining the pattern shown in Figure 5, $T$. davisiana davisiana and $L$. nigriniae, evolved after Time Slice 2. 


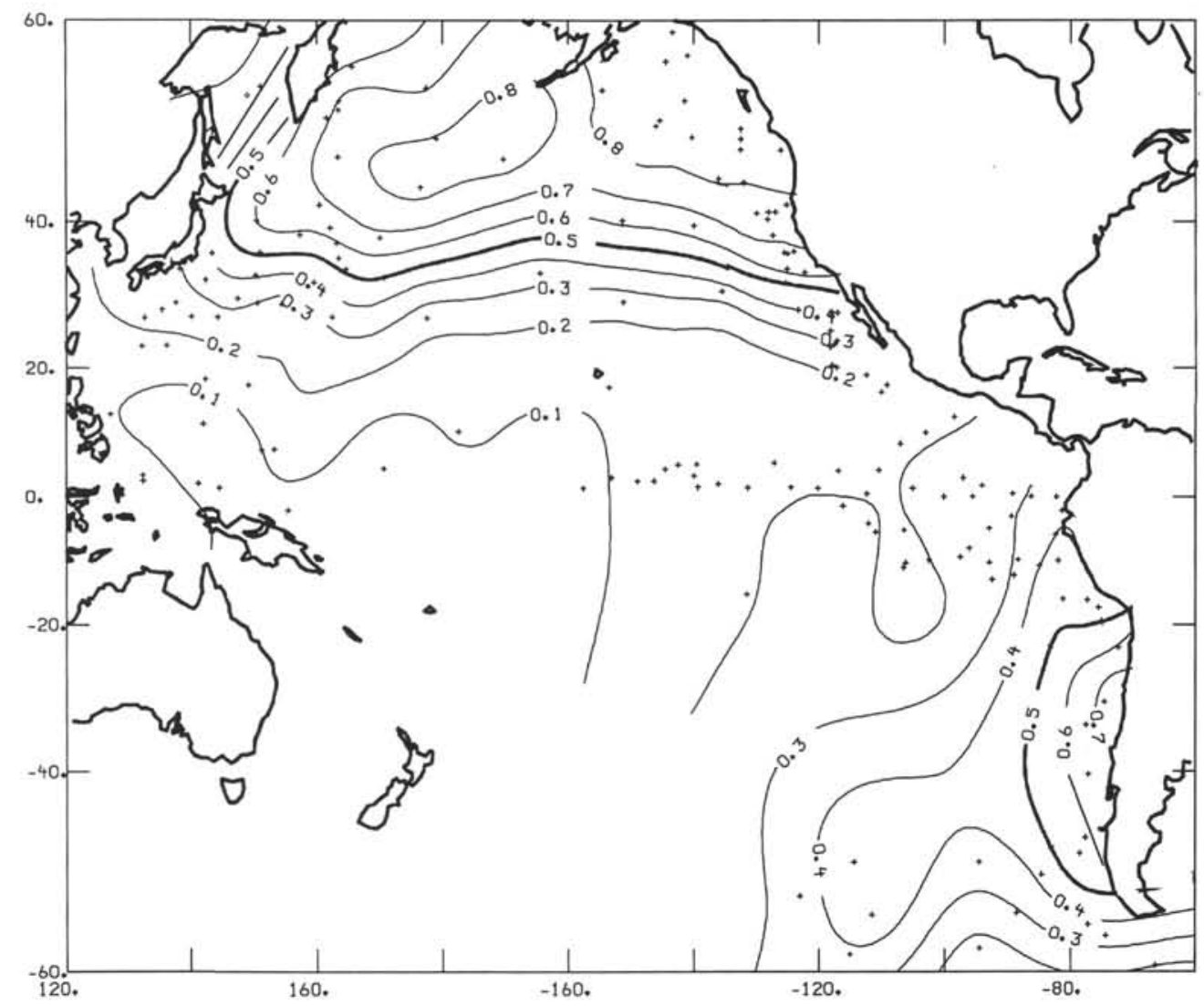

Figure 4. Distribution map of the factor loadings of the second (transitional) radiolarian assemblage extracted from analysis of surface sediments from the Pacific Ocean.

\section{RESULTS AND DISCUSSION}

\section{Time Slice 1 ( 2.3 Ma)}

In Table 4, we list the modern factor loadings and the communalities for the 2.3- to 2.5-Ma time slice. As noted above, the communality of a sample is a measure of how well the factors reconstruct the original sample. The high communalities of the time slice samples suggests that using modern-day radiolarian distributions to describe radiolarian population changes during the late Neogene is not unreasonable. The mean communality of samples from Time Slice 1 is 0.83 . This indicates that, for the 2.3- to 2.5-Ma time slice, more than $80 \%$ of the information contained in these data are described by the modern day assemblages.

In Figure 6, for the interval from 2.3 to $2.5 \mathrm{Ma}$, we plot the GRAPE density and the factor loadings for the tropical, transitional, and Peru assemblages in Sites 845 through 854. For Sites 846, 849, and 851 , we also plotted oxygen isotope data. The isotope data for Sites 846 and 849 are based on benthic foraminifers (Mix et al., this volume; Shackleton et al., this volume) and for Site 851 the data are from planktonic foraminifers (Ravelo and Shackleton, this volume).

The tropical and transitional assemblages are the most important assemblages during Time Slice 1. Recognizing that the resolution of these short radiolarian data sets differs from site to site, a consistent pattern of change is shared by most sites. In general, the GRAPE density minimum sampled in Time Slice 1 is characterized by a reduced level in the importance of the tropical assemblage and an increased importance in the transitional assemblage, whereas the GRAPE density maximum is characterized by an increased importance of the tropical assemblage. This pattern is most clearly demonstrated in Sites $846,847,850,852$, and 854 . In some sites, the changes in the transition fauna also has been paralleled by changes in the eastern boundary assemblage (e.g., Sites 847,850 , and 852 ).

Were we to have a perfectly uniform data set of time-correlative maxima and minima at each site, we would be able to make maps of the distribution of each assemblage at the times of these carbonate events. Instead, we can only contrast the assemblages found at the GRAPE (carbonate) minimum with those found at the GRAPE (carbonate) maximum at each site (Fig. 7). In Table 4, the samples used in the compilation have been identified. We selected samples by first identifying samples associated with a GRAPE minimum (GRAPE listings from the Leg 138 CD-ROM were used). Samples from the next youngest GRAPE maximum were then selected. If more than one sample was found to have similar GRAPE values, their factor values were averaged. By showing the change at each site from the GRAPE minimum to maximum, we are assuming that the relationship between surface oceanographic change (radiolarian assemblages) and change in carbonate deposition is linear and time invariant over the relatively short space of time represented by the data set. Although this assumption cannot be tested with this limited data set, we think that this is a conservative approach to exploring the differences in the surface ocean between times of carbonate maxima and minima.

The difference maps (Fig. 7) demonstrate the consistency of the pattern of change noted before. The GRAPE maximum is characterized by the increased importance (positive values) of the tropical assemblage (Fig. 7A) at nearly all sites, whereas the cooler water transition assemblage is more important during GRAPE minima (Fig. $7 \mathrm{~B}$; negative values). The largest response is seen in the southeastern sites (Sites 846 and 847), closest to the influence of the Peru Current (Figs. 7A and 7B), followed by a large response at the northernmost sites along the $110^{\circ} \mathrm{W}$ transect. Like the tropical assemblage, the 


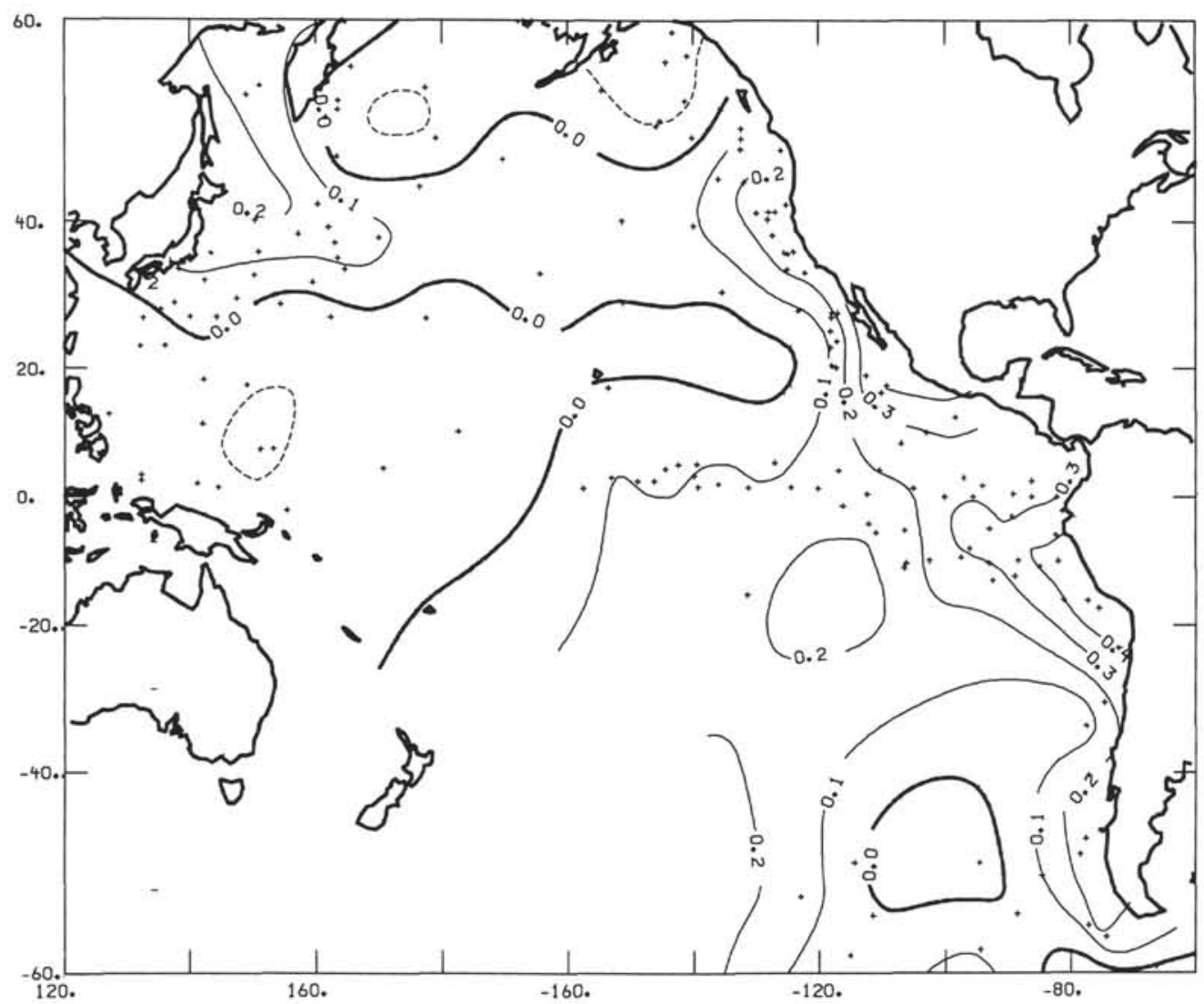

Figure 5. Distribution map of the factor loadings of the third (EBC) radiolarian assemblage extracted from analysis of surface sediments from the Pacific Ocean.

change in the relative importance of the transitional assemblage is of the same sign throughout the region (Fig. 7B).

The EBC assemblage, however, shows a different pattern between the eastern and western sites. In the east, this assemblage is more important during GRAPE minima much like the transitional assemblage. In the sites along $110^{\circ} \mathrm{W}$, the $\mathrm{EBC}$ assemblage generally shows slightly higher values during the GRAPE maxima. The most northerly site (854) and Site 852 may have been influenced by the North Pacific eastern boundary current feeding into the North Equatorial Current (NEC; Site 854) or by enhanced local divergence between the NEC and the North Equatorial Countercurrent (NECC; Site 852). Although we have assumed that the selected GRAPE events are synchronous, some of the observed differences may be the result of the slightly different time intervals sampled at each site. However, this would not explain the differences among Sites 852,851 , and 850 (Fig. 2A).

The pattern of change is consistent with the hypothesis that the low GRAPE (carbonate) events of the interval represented by Time Slice 1 are times of cooler, and possibly more productive, oceanographic conditions, as reflected by the decreased tropical assemblage and increased influence of transitional water species. This inference is supported by the isotope data available for Site 846 , which show a clear change from heavy to light isotopes, paralleling changes in the sediment from low to high GRAPE density (Fig. 6). The planktonic isotope data for Site 851 show a similar lightening, with increased warm-water radiolarian assemblages, although the pattern is less clear at Site 849 (Fig. 6).

The strong change in the EBC assemblage at Sites 847 and 846 suggests that advection from the Peru Current decreases during carbonate highs. The response of the more northerly sites of the $110^{\circ} \mathrm{W}$ transect may also reflect a decreased influence from the eastern North
Pacific. The response of the EBC species at sites nearer to the equator along $110^{\circ} \mathrm{W}$ shows relatively small differences among small numbers (loading); these differences may reflect more local wind-induced convergence and upwelling along the equatorial divergence during times of high carbonate. Thus, we may be seeing effects of local windinduced changes in circulation, combined with changes in the largescale circulation of the southeastern Pacific. This pattern of cooler oceanographic conditions during times of decreased carbonate content in the equatorial Pacific ocean is opposite to what is inferred from studies of late Pleistocene age sediments (e.g., Pisias and Rea, 1988).

\section{Time Slice $2(\sim 4.8 \mathrm{Ma})$}

In Table 5, we list the factor loadings and the communalities for the 4.7- to 5.0-Ma time slice, calculated from the factor matrix of Hagelberg and Pisias (1990). The mean communality in Time Slice 2 is 0.89 . This indicates that for this time slice, almost $90 \%$ of the information contained in the 35 selected extent species can be described by modern-day assemblages.

In Figure 8, we have plotted the GRAPE density and factor loadings for the tropical, western Pacific and EBC assemblage for the interval from 4.7 to $5.0 \mathrm{Ma}$ (as identified by Hagelberg and Pisias, 1990) at Sites 845 through 852 . For this time slice, the patterns shown in Figure 8 are less well defined compared to those for Time Slice 1. In a fashion similar to that used for Time Slice 1, we have averaged the factor loadings for the high and low GRAPE intervals sampled at each site and have shown the differences in Figure 9.

The change in the tropical assemblage during Time Slice 2 is similar to that during Time Slice 1. Most sites show a decrease in the importance of this assemblage at the GRAPE minima, with the largest changes found at the near-equator sites. Both the western Pacific and 

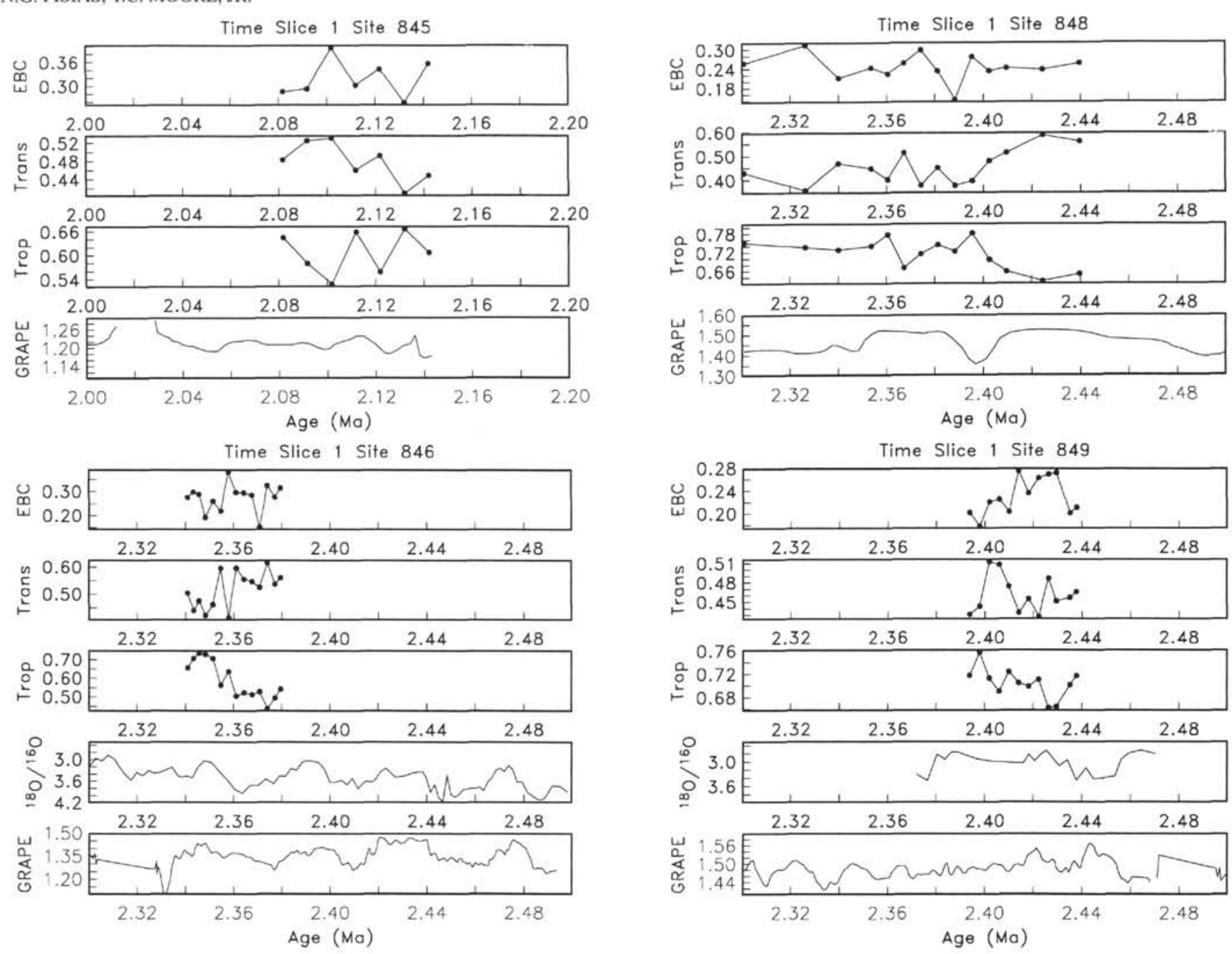

Time Slice 1 Site 847
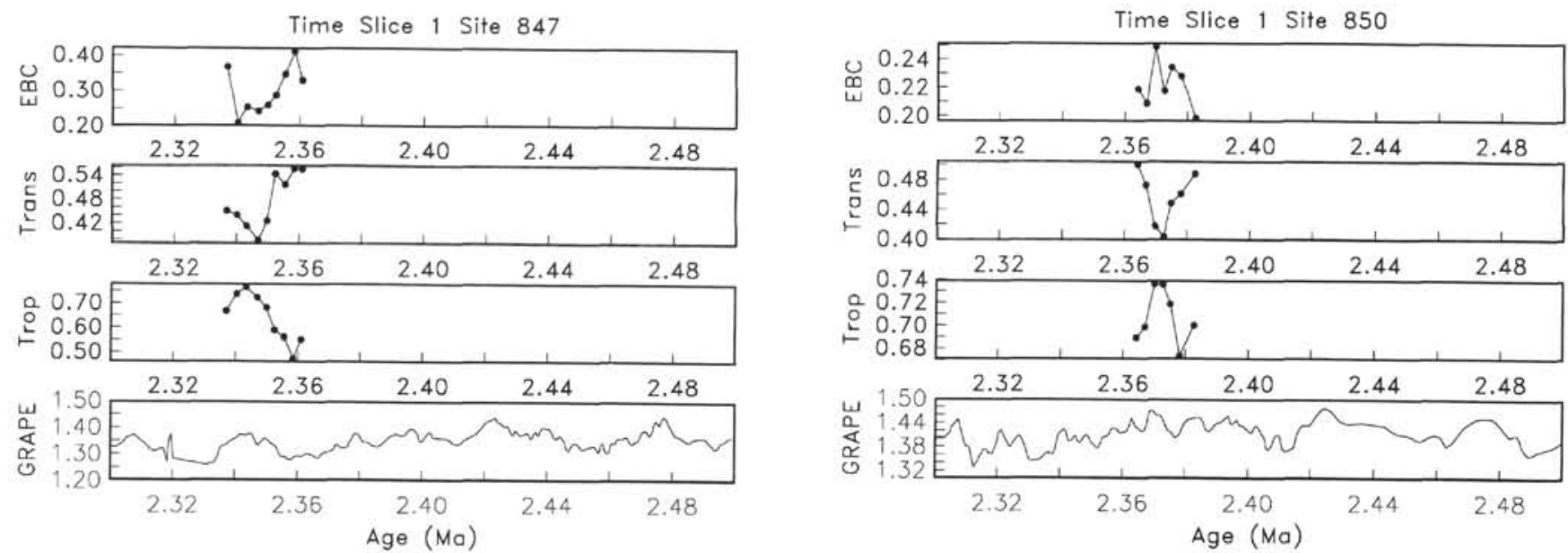

Figure 6. GRAPE density and factor loadings for the tropical, transitional, and EBC radiolarian assemblage in Sites 845 through 854 vs. age.

the $\mathrm{EBC}$ assemblage show similar patterns, with increased influence of these assemblages during GRAPE minima. In general, the parallel behavior of these later two assemblages may seem inconsistent, given the association with very warm conditions of the western Pacific assemblage vs. the cooler water association with the EBC assemblage. However, increased advection of near-surface waters and an enhanced ENSO cycle would produce stronger associations of these two assemblages, with the western Pacific assemblage being more important during El Niño events (Pisias et al., 1986), whereas the $\mathrm{EBC}$ assemblage would be important during the La Niña events.

An important difference in the patterns shown in Figures 7 and 9 is the opposite behavior of Site 847 relative to Site 846 . This may reflect circulation changes associated with open circulation between the tropical Atlantic and Pacific. Although Site 846 was dominated by the 


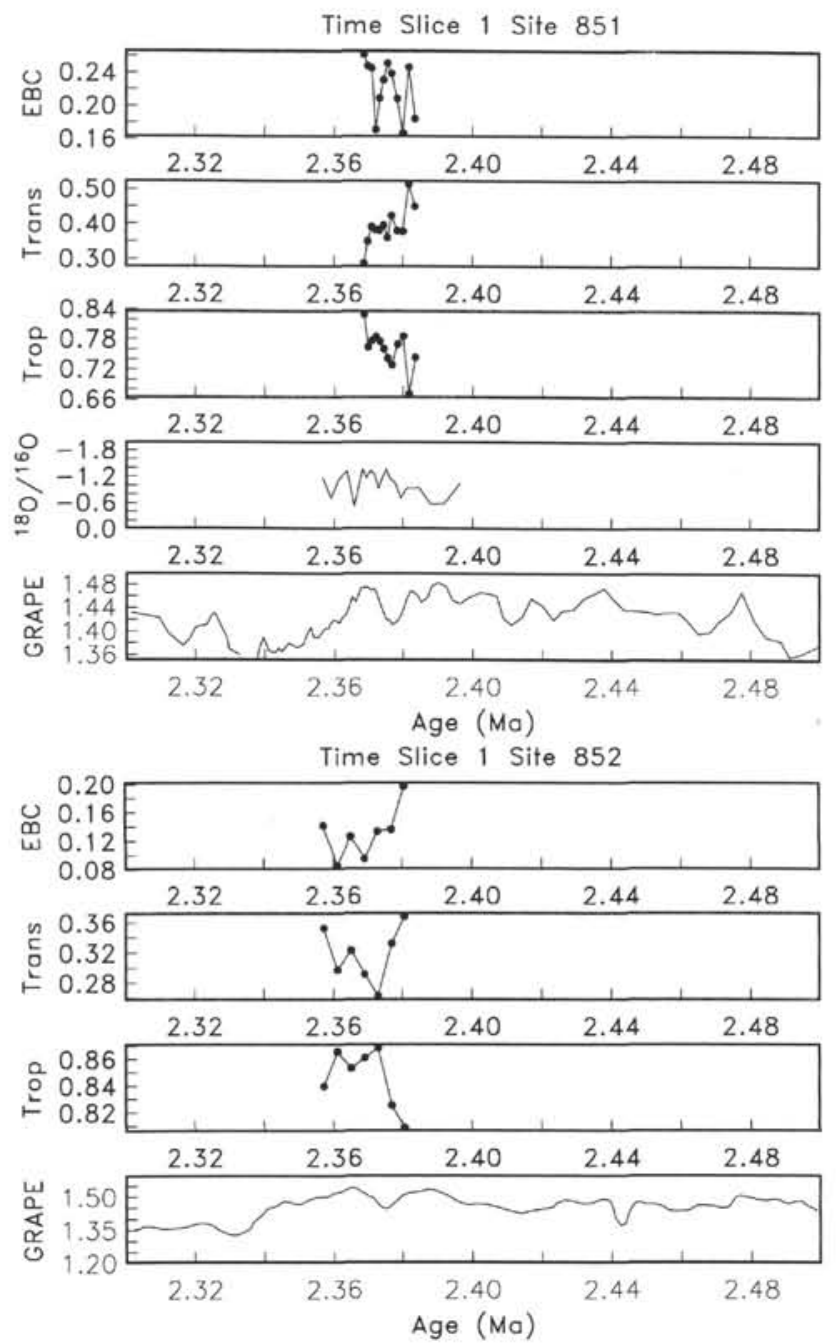

Figure 6 (continued).

paleo-Peru Current, Site 847 may be more influenced by the interaction of the tropical Pacific and Atlantic circulation. Radiolarian evolutionary events of the early Pliocene also show strong temporal diachrony between sites on and just south of the equator, suggesting strong oceanographic gradients in this region (Moore et al., 1993, and this volume). It is hoped that modeling results will help test this inference.

The results of Hovan (this volume) have important implications on possible circulation patterns during the older time slice of this study. Hovan shows that the Intertropical Convergence Zone (ITCZ) was positioned farther north before the early Pliocene. Thus, much of the equatorial circulation system was under the influence of the southern tradewinds. The seasonality of this system, then, would reflect only the season cycle of the southeastern trade winds rather than the effects of the seasonal changes in wind strength and the north-south migration of the ITCZ. Our results suggest that this wind circulation resulted in a much more zonal circulation (the out-of-phase behavior of Sites 846 and 847), combined with an enhanced seasonal cycle and/or enhanced El Niño cycle.

In contrast with the younger time slice, in which the tropical and transitional factors show consistent patterns of change throughout the study area during intervals of high and low GRAPE density, the older time slice shows marked zonal shifts in these patterns. During times of high GRAPE density, the tropical factor dominated the region just north of the equator (presumably the NECC). During times of low GRAPE density, the tropical factor dominated in regions of the SEC and NEC, whereas the western Pacific factor was most important in the region just north of the equator and the Peru factor was most important
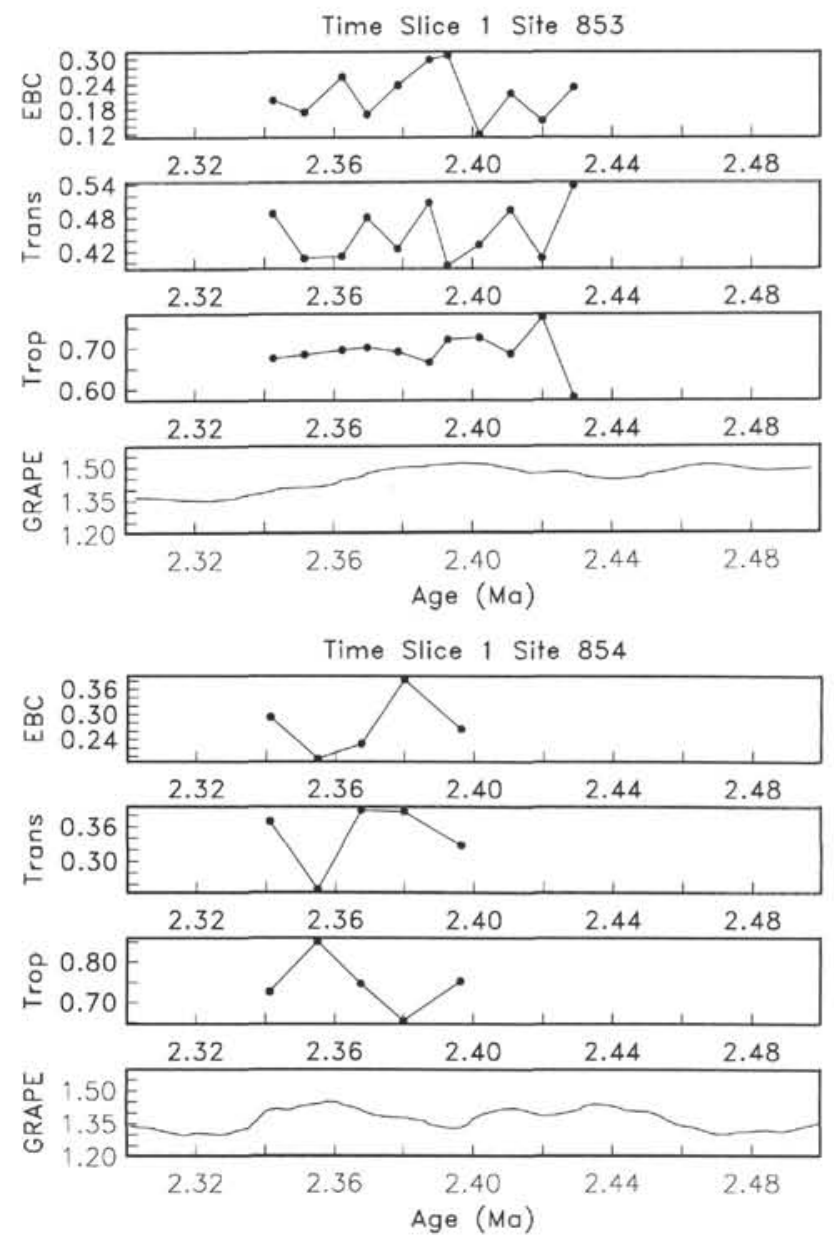

very close to the equator, particularly in the east (Site 847). Again, this points to stronger zonal advection during times of low carbonate.

\section{CONCLUSIONS}

These preliminary results suggest answers to the questions we have posed for this study. A systematic response appears to be found in the radiolarian assemblages of earliest and late Pliocene ages that can be associated with changes in carbonate deposition in the eastern equatorial Pacific. These data are consistent with the hypothesis that low carbonate intervals are related to cooler water conditions and possibly enhanced opal accumulation relative to carbonate accumulation. Times of increased carbonate content are associated with inferred warmer oceanographic conditions: dominance of the tropical assemblage during Time Slice 1 and combined importance of tropical and western Pacific factors in Time Slice 2.

The latest studies on carbonate and biogenic silica deposition in the eastern equatorial Pacific seem to indicate that, at least in this region, the high rate of carbonate production during glacial intervals is the controlling factor in giving rise to the pattern of carbonate-rich sediments in the glacials and carbonate-poor sediments in the interglacials of the late Pleistocene (Archer 1991a, 1991b). Comparison of the radiolarian assemblages and the intervals of high and low carbonate in the 2.3- and 4.8-Ma time slices studied here seem to suggest that both before and after the closing of the Isthmus of Panama, high carbonate intervals are associated with the greater dominance of the tropical radiolarian assemblage - an assemblage thought to be indicative of 
N.G. PISIAS, T.C. MOORE, JR.
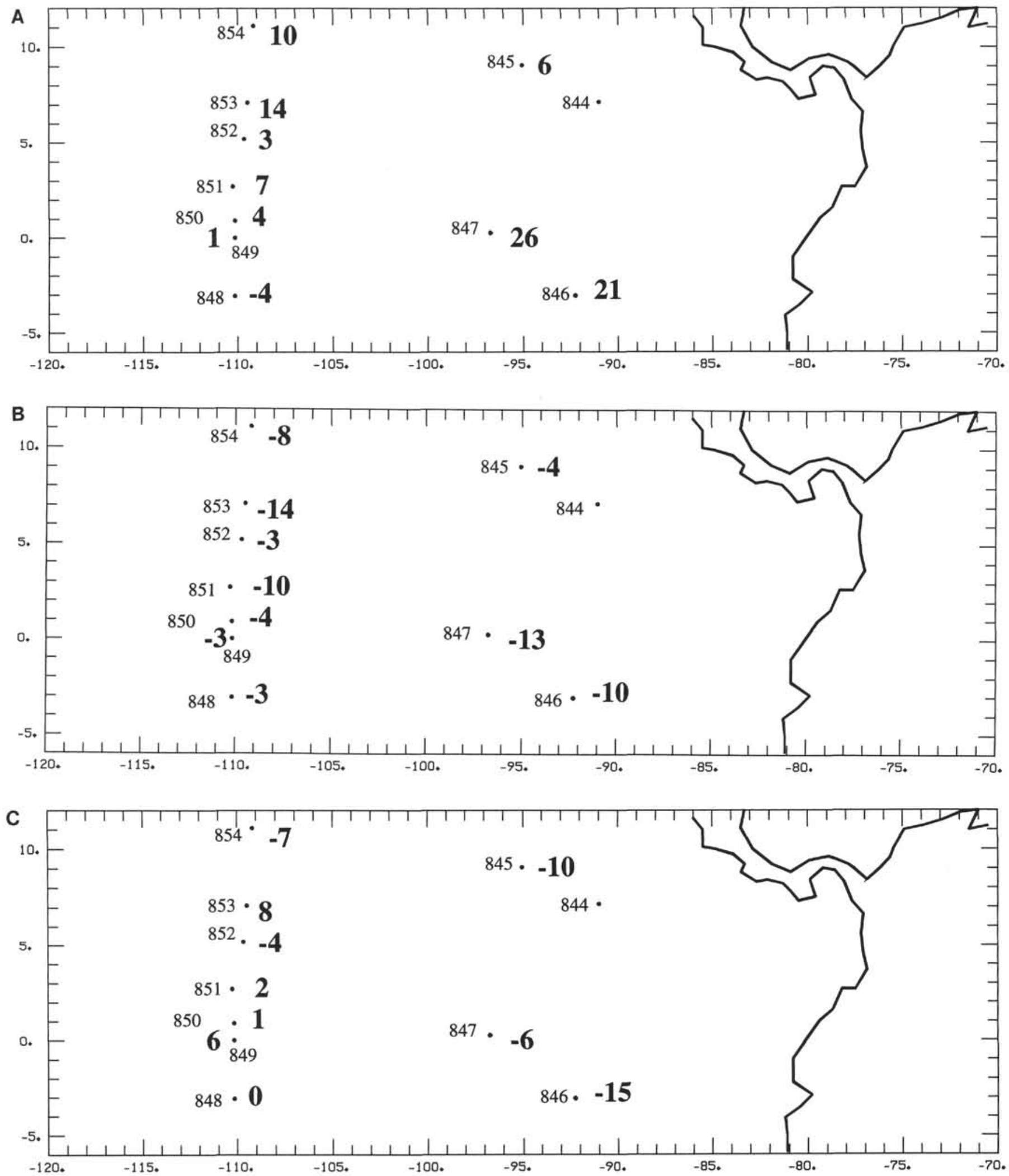

Figure 7. Difference between the mean loadings of each radiolarian assemblage $(\times 100)$ estimated during a GRAPE maxima minus the mean during a GRAPE minima during Time Slice 1. A. Tropical assemblage. B. Transitional assemblage. C. EBC assemblage. 

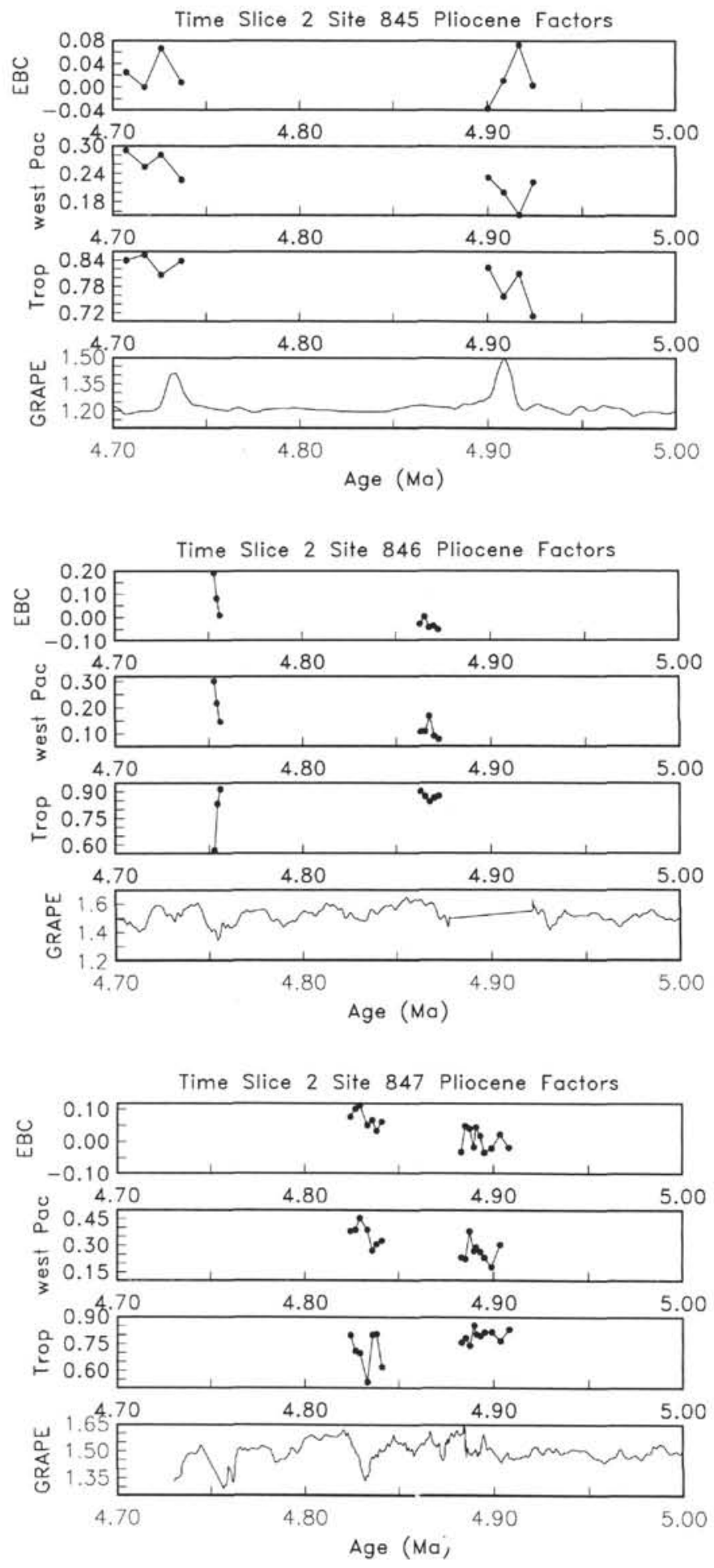
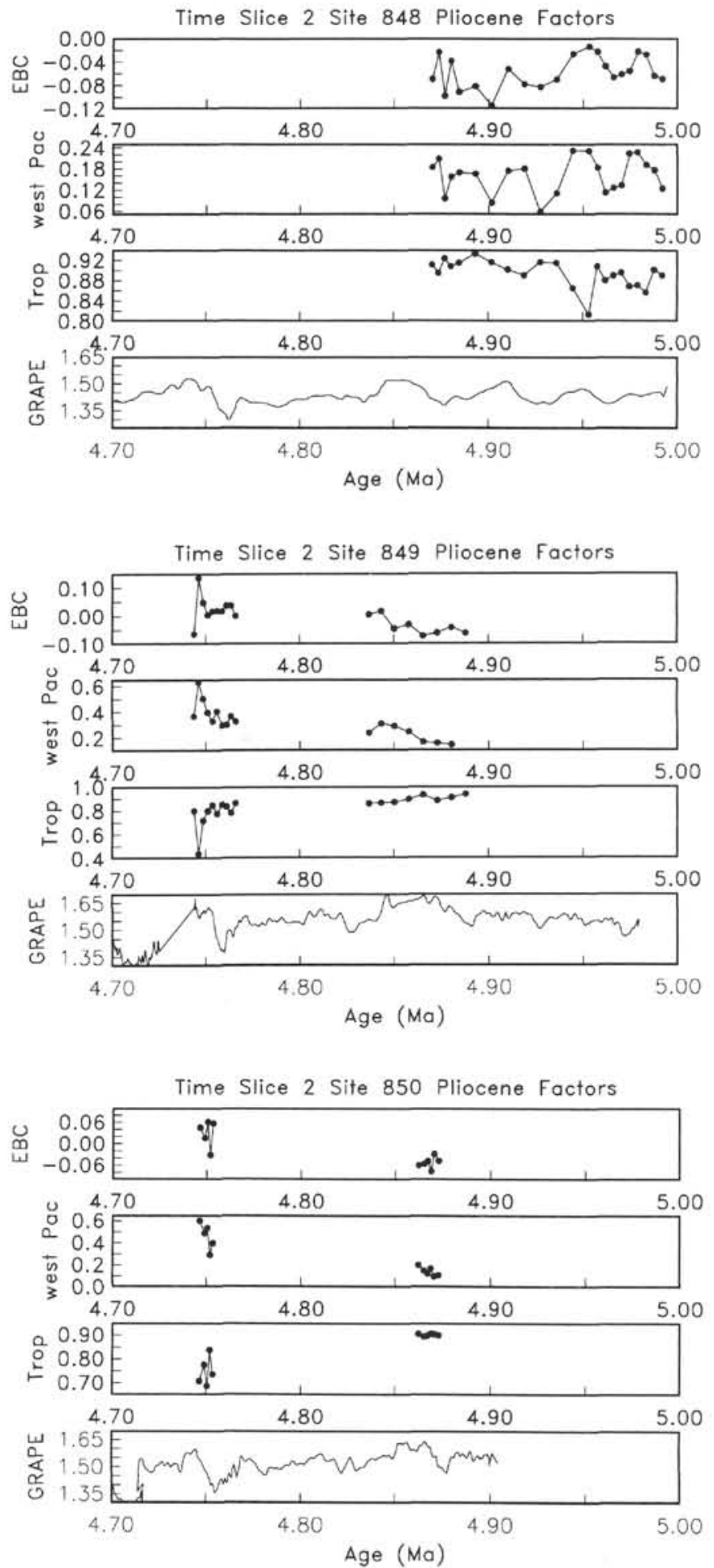

Figure 8. GRAPE density and factor loadings for the tropical, western Pacific, and EBC assemblages. Factors are based on an analysis of Pliocene sediments from the equatorial Pacific (Hagelberg and Pisias, 1990). Data are for Sites 845 through 854.

relatively less productive near-surface waters. Thus, it appears that either carbonate preservation is a stronger control on the Pliocene stratigraphic record, or that variation in the flux of biogenic silica controlled carbonate percentages and the GRAPE record.

The geographic patterns defined from these data are preliminary and await more detailed time-series analysis within each time slice. However, there is a suggestion that the largest responses are found in the southeastern sites of the study and in sites within the zone of equatorial divergence. This is consistent with the inferences made based on the variability of carbonate deposition in the Leg 138 drill sites (Hagelberg et al., this volume). In their study, Hagelberg et al. (this volume) noted an out-of-phase relationship between the easternmost sites and the $110^{\circ} \mathrm{W}$ sites. This pattern was also seen in the radiolarian analysis, especially during the earliest Pliocene. Although 
Time Slice 2 Site 851 Pliocene Factors

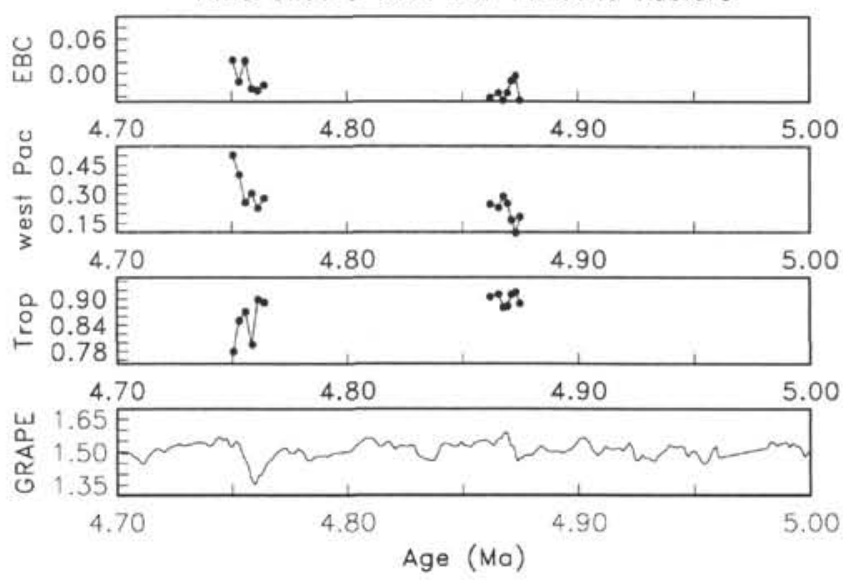

Figure 8 (continued).

Table 3. Factor score matrix from factor analysis of $\mathbf{4 1}$ radiolarian species found in recent sediments of the Pacific.

\begin{tabular}{|c|c|c|c|c|}
\hline & Species & Tropical & Transitional & $\begin{array}{c}\text { Eastern } \\
\text { boundary }\end{array}$ \\
\hline 1 & S1 & -0.0328 & 0.0717 & 0.1661 \\
\hline 2 & S1A & 0.1040 & -0.0302 & -0.0071 \\
\hline 3 & S7 & -0.0070 & 0.2169 & 0.1116 \\
\hline 4 & S8 & -0.0283 & 0.2953 & 0.0616 \\
\hline 5 & S10 & 0.0402 & 0.3611 & 0.0352 \\
\hline 6 & $\mathrm{~S} 12$ & 0.1731 & -0.0582 & -0.0087 \\
\hline 7 & $\mathrm{~S} 13$ & 0.1337 & -0.0070 & -0.0260 \\
\hline 8 & $\mathrm{~S} 14$ & 0.2074 & -0.0136 & 0.0306 \\
\hline 9 & S17 & 0.1110 & 0.1160 & -0.0572 \\
\hline 10 & S18 & 0.0876 & -0.0183 & 0.0106 \\
\hline II & S23 & 0.3878 & -0.1016 & -0.0627 \\
\hline 12 & S24 & 0.0672 & 0.4156 & 0.1890 \\
\hline 13 & S29 & 0.0641 & 0.2656 & -0.0292 \\
\hline 14 & S30 & 0.4815 & 0.0149 & 0.0607 \\
\hline 15 & S36A & 0.0621 & -0.0249 & -0.0031 \\
\hline 16 & $\mathrm{~S} 36 \mathrm{C}$ & 0.1176 & -0.0528 & -0.0048 \\
\hline 17 & S40 & 0.1547 & -0.0592 & -0.0049 \\
\hline 18 & $\mathrm{~S} 43$ & -0.0151 & 0.0376 & 0.3174 \\
\hline 19 & S47 & 0.0122 & 0.2369 & 0.1648 \\
\hline 20 & S48 & 0.0365 & 0.1458 & -0.0151 \\
\hline 21 & S54 & 0.5818 & 0.0294 & 0.0492 \\
\hline 22 & NI & 0.1118 & -0.0288 & -0.0153 \\
\hline 23 & $\mathrm{~N} 2$ & 0.0621 & -0.0110 & -0.0094 \\
\hline 24 & N5 & -0.0098 & 0.0608 & -0.0402 \\
\hline 25 & N7 & 0.0359 & -0.0511 & -0.0046 \\
\hline 26 & N9 & 0.1879 & -0.0443 & -0.0150 \\
\hline 27 & $\mathrm{~N} 14$ & 0.0635 & -0.0673 & 0.2476 \\
\hline 28 & N15 & -0.0024 & 0.0925 & 0.0072 \\
\hline 29 & N18 & 0.0261 & -0.0008 & 0.0046 \\
\hline 30 & N24 & -0.0082 & 0.0299 & 0.0067 \\
\hline 31 & $\mathrm{~N} 27$ & 0.1434 & -0.0112 & -0.0207 \\
\hline 32 & $\mathrm{~N} 29$ & -0.0040 & 0.1400 & 0.0075 \\
\hline 33 & N32 & 0.0243 & -0.0309 & 0.0645 \\
\hline 34 & N33 & -0.0390 & 0.3948 & -0.0927 \\
\hline 35 & N35 & -0.0617 & 0.1069 & 0.0904 \\
\hline 36 & $\mathrm{~N} 35 \mathrm{~A}$ & -0.0038 & 0.0924 & 0.0120 \\
\hline 37 & N38 & -0.0064 & 0.2192 & -0.0263 \\
\hline 38 & $\mathrm{~N} 40$ & 0.0912 & 0.2335 & -0.0157 \\
\hline 39 & $\mathrm{~N} 42$ & 0.0891 & 0.0122 & -0.0221 \\
\hline 40 & $\mathrm{~N} 43$ & 0.0055 & 0.1653 & -0.0898 \\
\hline 41 & N46 & -0.0386 & -0.1476 & 0.8249 \\
\hline
\end{tabular}

these results are preliminary, they demonstrate the importance of having adequate geographic coverage of sites in an area of complex oceanography such as the eastern equatorial Pacific. The importance of this spatial coverage is emphasized by the distinct differences in the mapped patterns of factor dominance during Time Slice 1, compared with that of Time Slice 2. The older time slice, representing a time before the closing of the Isthmus of Panama, shows a sharp zonality of patterns, presumably associated with the more zonal character of equatorial circulation. After the closing of the isthmus, the shifts in faunal patterns between times of high and low carbonates were characterized by shifts in the dominance of the tropical and transitional assemblages, respectively, throughout the region.

\section{REFERENCES}

Adelseck, C.G., Jr., and Anderson, T.F., 1978. The late Pleistocene record of productivity fluctuations in the eastern equatorial Pacific Ocean. Geology, 6:388-391.

Alexandrovich, J.M., 1989. Radiolarian biostratigraphy of ODP Leg 111, Site 677, eastern equatorial Pacific, late Miocene through Pleistocene. In Becker, K., Sakai, H., et al., Proc. ODP, Sci. Results, 111: College Station, TX (Ocean Drilling Program), 245-262.

Archer, D.E., 1991a. Equatorial Pacific calcite preservation cycles: production or dissolution? Paleoceanography, 6:561-571.

, 1991b. Modeling the calcite lysocline. J. Geophys. Res., 96:1703717050.

Arrhenius, G., 1952. Sediment cores from the east Pacific. Rep. Swed. DeepSea Exped. 1947-1948, 5:189-201.

Benson, R.N., 1966. Recent Radiolaria from the Gulf of California [Ph.D. dissert.]. Univ. of Minnesota.

Berger, W.H., 1973. Deep-sea carbonates: Pleistocene dissolution cycles. J. Foraminiferal Res., 3:187-195.

Caulet, J.-P., and Nigrini, C., 1988. The genus Pterocorys (Radiolaria) from the tropical late Neogene of the Indian and Pacific oceans. Micropaleontology, 34:217-235.

Farrell, J.W., and Prell, W.L., 1989. Climatic change and $\mathrm{CaCO}_{3}$ preservation: an 800,000 year bathymetric reconstruction from the central equatorial Pacific Ocean. Paleoceanography, 4:447-466.

- 1991. Pacific $\mathrm{CaCO}_{3}$ preservation and $\delta^{18} \mathrm{O}$ since 4 Ma: paleoceanic and paleoclimatic implications. Paleoceanography, 6:485-498.

Hagelberg, T.K., and Pisias, N., 1990. Nonlinear response of Pliocene climate to orbital forcing: evidence from the eastern equatorial Pacific. Paleoceanography, 5:595-617.

Hays, P.E., Pisias, N.G., and Roelofs, A.K., 1989. Paleoceanography of the eastern equatorial Pacific during the Pliocene: a high-resolution radiolarian study. Paleoceanography, 4:57-73.

Imbrie, J., and Kipp, N.G., 1971. A new micropaleontological method for quantitative paleoclimatology: application to a late Pleistocene Caribbean core. In Turekian, K.K. (Ed.), The Late Cenozoic Glacial Ages: New Haven (Yale Univ. Press), 71-181.

Kling, S.A., 1973. Radiolaria from the eastern North Pacific, Deep Sea Drilling Project, Leg 18. In Kulm, L.D., von Huene, R., et al., Init. Repts. DSDP, 18: Washington (U.S. Govt. Printing Office), 617-671.

\footnotetext{
- Abbreviations for names of organizations and publication titles in ODP reference lists follow the style given in Chemical Abstracts Service Source Index (published by American Chemical Society).
} 
Knoll, A., and Johnson, D.A., 1975. Late Pleistocene evolution of the collosphaerid radiolarian Buccinosphaera invaginata Haeckel. Micropaleontology, 21:60-68.

Molina-Cruz, A., 1977. Radiolarian assemblages and their relationship to the oceanography of the subtropical southeastern Pacific. Mar. Micropaleontol., 2:315-352.

Moore, T.C., Jr., 1978. The distribution of radiolarian assemblages in the modern and ice-age Pacific. Mar. Micropaleontol., 2:229-266.

Moore, T.C., Jr., Burckle, L.H., Geitzenauer, K., Luz, B., Molina-Cruz, A., Robertson, J.H., Sachs, H., Sancetta, C., Thiede, J., Thompson, P., and Wenkam, C., 1980. The reconstruction of sea surface temperatures in the Pacific Ocean of 18,000 B.P. Mar. Micropaleontol., 5:215-247.

Moore, T.C., Jr., Shackleton, N.J., and Pisias, N.G., 1993. Paleoceanography and the diachrony of radiolarian events in the eastern equatorial Pacific. Paleoceanography, 8:567-586.

Morley, J.J., 1980. Analysis of the abundance variations of the subspecies of Cycladophora davisiana. Mar. Micropaleontol., 5:205-214.

Nigrini, C., and Lombari, G., 1984. A Guide to Miocene Radiolaria. Spec. Publ. Cushman Found. Foraminiferal Res., 22.

Nigrini, C., and Moore, T.C., Jr., 1979. A Guide to Modern Radiolaria. Spec. Publ. Cushman Found. Foraminiferal Res., 16.

Pisias, N.G., Murray, D.W., and Roelofs, A.K., 1986. Radiolarian and silicoflagellate response to oceanographic changes associated with the 1983 El Nino. Nature, 320:259-262.

Riedel, W.R., 1953. Mesozoic and late Tertiary Radiolaria of Rotti. J. Paleontol., 27:805-813.
Robertson, J.H., 1975. Glacial to interglacial oceanographic changes in the northwest Pacific, including a continuous record of the last 400,000 years [Ph.D. dissert.]. Columbia Univ., New York.

Roelofs, A., and Pisias, N.G., 1986. Revised technique for preparing quantitative radiolarian slides from deep-sea sediments. Micropaleontology, 24:182-185.

Romine, K., and Moore, T.C., Jr., 1981. Radiolarian assemblage distributions and paleoceanography of the Eastern Equatorial Pacific Ocean during the last 127,000 years. Palaeogeogr., Palaeoclimatol., Palaeoecol., 35:281-314.

Sachs, H., 1973. North Pacific radiolarian assemblages and their relationship to oceanographic parameters. J. Quat. Res., 3:73-88.

Schramm, C.T., 1985. Implications of radiolarian assemblages for the Late Quaternary paleoceanography of the eastern equatorial Pacific. Quat. Res. (N.Y.), 24:204-218.

Shackleton, N.J., and Shipboard Scientific Party, 1992. Sedimentation rates: toward a GRAPE density stratigraphy for Leg 138 carbonate sections. In Mayer, L., Pisias, N., Janecek, T., et al., Proc. ODP, Init. Repts., 138 (Pt. 1): College Station, TX (Ocean Drilling Program), 87-91.

Takahashi, K., and Honjo, S., 1981. Vertical flux of Radiolaria: a taxonquantitative sediment trap study from the western tropical Atlantic. Micropaleontology, 27:140-190.

Date of initial receipt: 9 March 1993

Date of acceptance: 15 November 1993

Ms 138SR-124 

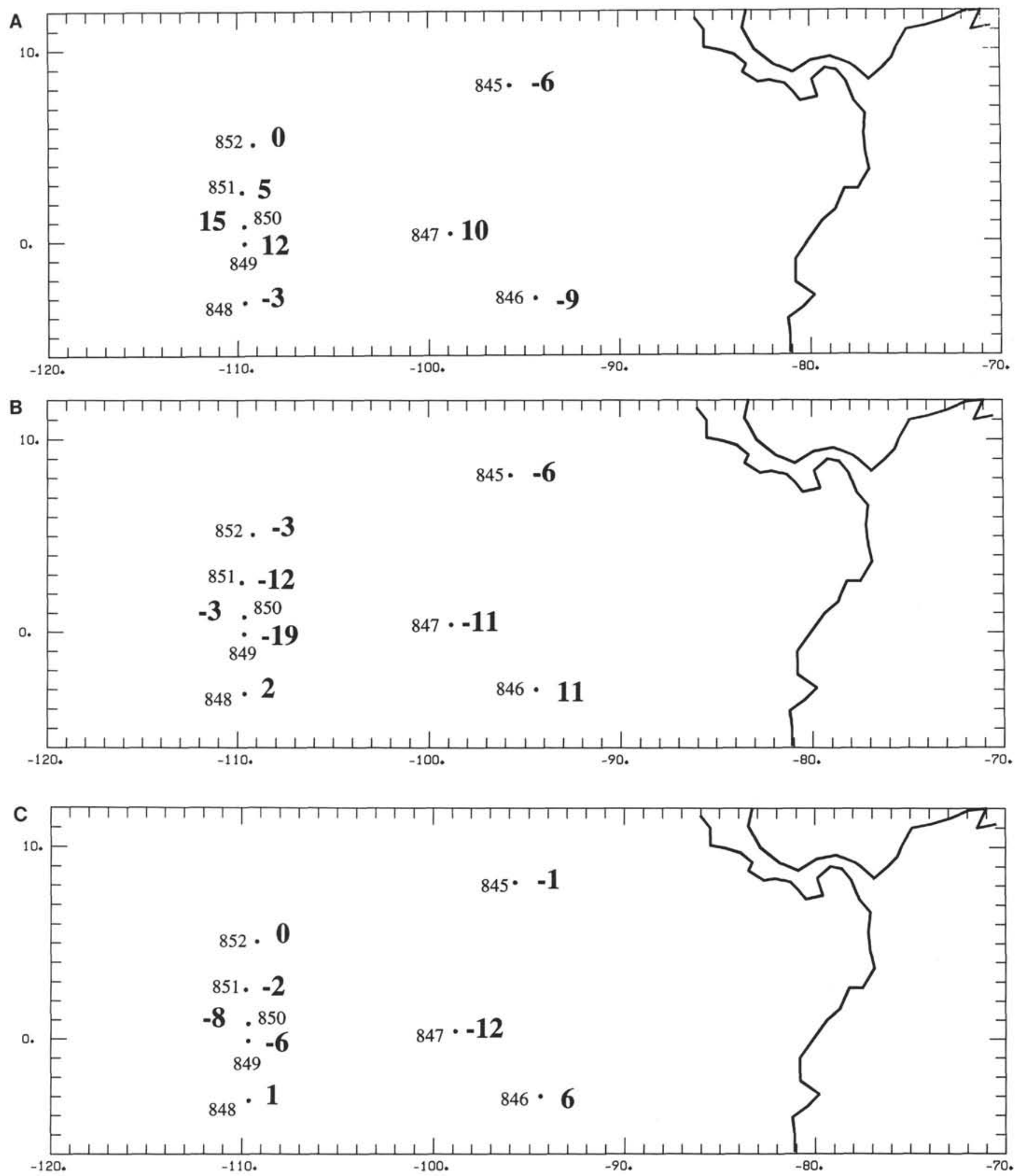

Figure 9. Difference between the mean loadings of each radiolarian assemblage $(\times 100)$ estimated during a GRAPE maxima minus the mean during a GRAPE minima during Time Slice 2. A. Tropical assemblage. B. Western Pacific assemblage. C. EBC assemblage. 
Table 4. Factor-loading matrix estimate for the Time Slice 1 samples listed in Table 1.

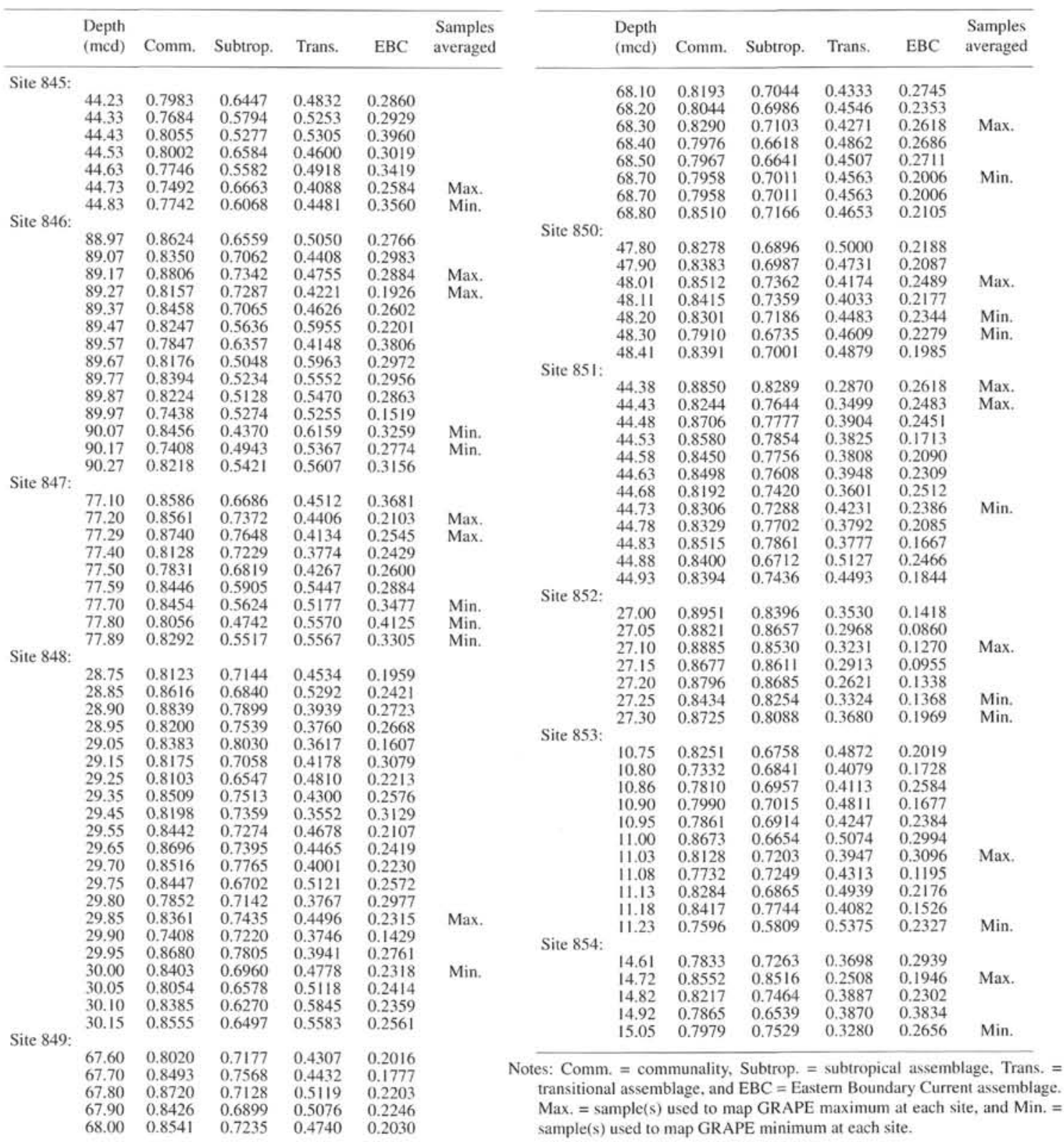


Table 5. Factor-loading matrix estimate for Time Slice 2 samples listed in Table 1.

\begin{tabular}{|c|c|c|c|c|c|c|c|c|c|c|c|}
\hline & $\begin{array}{l}\text { Depth } \\
\text { (mcd) }\end{array}$ & Comm. & Trop. & West Pac. & EBC & & $\begin{array}{l}\text { Depth } \\
\text { (mcd) }\end{array}$ & Comm. & Trop. & West Pac. & EBC \\
\hline \multirow[t]{9}{*}{ Site 845: } & & & & & & & 143.60 & 0.9031 & 0.7974 & 0.3963 & 0.0044 \\
\hline & 70.81 & 0.9392 & 0.8392 & 0.2897 & 0.0255 & & 143.80 & 0.8979 & 0.8468 & 0.3295 & 0.0167 \\
\hline & 70.91 & 0.9476 & 0.8517 & 0.2538 & -0.0002 & & 144.00 & 0.8915 & 0.7741 & 0.4063 & 0.0189 \\
\hline & 71.00 & 0.9250 & 0.8056 & 0.2802 & 0.0661 & & 144.20 & 0.9083 & 0.8501 & 0.2962 & 0.0170 \\
\hline & 71.11 & 0.9293 & 0.8377 & 0.2265 & 0.0085 & & 144.40 & 0.9030 & 0.8350 & 0.3017 & 0.0375 \\
\hline & 72.31 & 0.9502 & 0.8227 & 0.2322 & -0.0362 & & 144.60 & 0.8608 & 0.7810 & 0.3661 & 0.0377 \\
\hline & 72.41 & 0.9309 & 0.7576 & 0.2007 & 0.0112 & & 144.80 & 0.8940 & 0.8630 & 0.3262 & 0.0014 \\
\hline & 72.51 & 0.8868 & 0.8096 & 0.1527 & 0.0725 & & 147.40 & 0.8970 & 0.8592 & 0.2392 & 0.0045 \\
\hline & 72.60 & 0.8972 & 0.7135 & 0.2226 & 0.0038 & & 147.60 & 0.9171 & 0.8642 & 0.3093 & 0.0166 \\
\hline \multirow{9}{*}{ Site 846 : } & & & & & & & 147.80 & 0.9348 & 0.8685 & 0.2883 & -0.0455 \\
\hline & 185.54 & 0.6913 & 0.5701 & 0.3010 & 0.1899 & & 148.00 & 0.9308 & 0.8942 & 0.2445 & -0.0312 \\
\hline & 185.64 & 0.8529 & 0.8321 & 0.2159 & 0.0817 & & 148.20 & 0.9504 & 0.9302 & 0.1651 & -0.0717 \\
\hline & 185.74 & 0.9159 & 0.9139 & 0.1460 & 0.0100 & & 148.40 & 0.9386 & 0.8813 & 0.1585 & -0.0611 \\
\hline & 190.00 & 0.8741 & 0.9018 & 0.1079 & -0.0265 & & 148.60 & 0.9171 & 0.9076 & 0.1453 & -0.0419 \\
\hline & 190.10 & 0.8428 & 0.8732 & 0.1086 & 0.0028 & & 148.80 & 0.9421 & 0.9375 & 0.0947 & -0.0619 \\
\hline & 190.20 & 0.8244 & 0.8441 & 0.1673 & -0.0421 & Site 850: & & & & & \\
\hline & 190.30 & 0.8812 & 0.8646 & 0.0913 & -0.0364 & & 107.36 & 0.9184 & 0.7081 & 0.6013 & 0.0453 \\
\hline & 190.40 & 0.8716 & 0.8757 & 0.0790 & -0.0513 & & 107.53 & 0.9356 & 0.7759 & 0.4873 & 0.0158 \\
\hline \multirow{19}{*}{ Site 847: } & & & & & & & 107.63 & 0.8891 & 0.6871 & 0.5353 & 0.0615 \\
\hline & 165.02 & 0.7894 & 0.7559 & 0.2334 & -0.0318 & & 107.72 & 0.9164 & 0.8380 & 0.2907 & -0.0313 \\
\hline & 165.22 & 0.7935 & 0.7809 & 0.2243 & 0.0490 & & 107.82 & 0.8854 & 0.7360 & 0.3985 & 0.0565 \\
\hline & 165.42 & 0.8346 & 0.7388 & 0.3785 & 0.0410 & & 112.07 & 0.9241 & 0.9062 & 0.2007 & -0.0591 \\
\hline & 165.62 & 0.8920 & 0.8521 & 0.2678 & -0.0169 & & 112.17 & 0.9371 & 0.8947 & 0.1496 & -0.0553 \\
\hline & 165.73 & 0.8545 & 0.8026 & 0.2884 & 0.0455 & & 112.27 & 0.9212 & 0.8982 & 0.1235 & -0.0480 \\
\hline & 165,93 & 0.8593 & 0.7936 & 0.2628 & 0.0183 & & 112.37 & 0.9274 & 0.9067 & 0.1696 & -0.0761 \\
\hline & 166.13 & 0.8403 & 0.8135 & 0.2304 & -0.0343 & & 112.47 & 0.9248 & 0.9039 & 0.0968 & -0.0284 \\
\hline & 166.33 & 0.8640 & 0.8155 & 0.1788 & -0.0205 & & 112.62 & 0.9049 & 0.8993 & 0.1102 & -0.0469 \\
\hline & 166.53 & 0.8373 & 0.7646 & 0.3036 & 0.0222 & & & & & & \\
\hline & 166.73 & 0.8447 & 0.8290 & 0.0822 & -0.0178 & ite 851: & 90.92 & & & & \\
\hline & 160.14 & 0.9035 & 0.7969 & 0.3805 & 0.0770 & & $\begin{array}{l}90.92 \\
91.02\end{array}$ & 0.9160 & 0.7793 & 0.5019 & 0.0237 \\
\hline & 160.34 & 0.7988 & 0.7090 & 0.3865 & 0.1025 & & 91.02 & 0.9546 & 0.8500 & 0.4000 & -0.0139 \\
\hline & 160.51 & 0.8685 & 0.6966 & 0.4533 & 0.1133 & & 91.12 & 0.9014 & 0.8702 & 0.2576 & 0.0224 \\
\hline & 160.80 & 0.7737 & 0.5363 & 0.3883 & 0.0517 & & 91.22 & 0.8699 & 0.7955 & 0.3045 & -0.0262 \\
\hline & 160.99 & 0.8638 & 0.7986 & 0.2710 & 0.0668 & & 91.32 & 0.9341 & 0.8984 & 0.2276 & -0.0295 \\
\hline & 161.15 & 0.8911 & 0.8038 & 0.3068 & 0.0336 & & 91.42 & 0.9392 & 0.8916 & 0.2800 & -0.0198 \\
\hline & 161.36 & 0.7843 & 0.6203 & 0.3261 & 0.0619 & & 93.82 & 0.9409 & 0.9044 & 0.2478 & -0.0425 \\
\hline & & & & & & & 93.92 & 0.9418 & 0.9102 & 0.2296 & 0.0338 \\
\hline \multirow{22}{*}{ Site 848: } & & 09443 & 00114 & & & & 94.02 & 0.9320 & 0.8802 & 0.2872 & -0.0462 \\
\hline & 46.05 & 0.9443 & 0.9114 & 0.1843 & -0.0692 & & 94.12 & 0.9302 & 0.8829 & 0.2519 & -0.0338 \\
\hline & 46.10 & 0.9280 & 0.8949 & 0.2083 & -0.0235 & & 94.22 & 0.9331 & 0.9100 & 0.1664 & -0.0129 \\
\hline & 46.15 & 0.9238 & 0.9237 & 0.0963 & -0.0977 & & 94.32 & 0.9161 & 0.9148 & 0.1006 & -0.0039 \\
\hline & 46.20 & 0.9155 & 0.9080 & 0.1576 & -0.0383 & & 94.42 & 0.9238 & 0.8890 & 0.1831 & -0.0464 \\
\hline & 46.25 & $\begin{array}{l}0.9319 \\
0.9446\end{array}$ & 0.9147 & $\begin{array}{l}0.1699 \\
0.1662\end{array}$ & -0.0914 & Site 852 & & & & & \\
\hline & $\begin{array}{l}46.35 \\
46.45\end{array}$ & $\begin{array}{l}0.9446 \\
0.9340\end{array}$ & 0.9332 & 0.1662 & -0.0812 & Site 852: & & & & & \\
\hline & $\begin{array}{l}46.45 \\
46.55\end{array}$ & $\begin{array}{l}0.9340 \\
0.9259\end{array}$ & $\begin{array}{l}0.9164 \\
0.9017\end{array}$ & $\begin{array}{l}0.0848 \\
0.1746\end{array}$ & -0.1142 & & $\begin{array}{l}53.75 \\
53.80\end{array}$ & 0.9305 & 0.8231 & 0.4121 & 0.0157 \\
\hline & $\begin{array}{l}46.53 \\
46.65\end{array}$ & $\begin{array}{l}0.9259 \\
0.8894\end{array}$ & $\begin{array}{l}0.9017 \\
0.8908\end{array}$ & $\begin{array}{l}0.1746 \\
0.1809\end{array}$ & $\begin{array}{l}-0.0517 \\
-0.0778\end{array}$ & & $\begin{array}{l}53.80 \\
53.85\end{array}$ & $\begin{array}{l}0.9384 \\
0.9299\end{array}$ & $\begin{array}{l}0.8740 \\
0.8522\end{array}$ & $\begin{array}{l}0.3069 \\
0.3727\end{array}$ & $\begin{array}{l}-0.0293 \\
-0.0052\end{array}$ \\
\hline & 46.75 & 0.9131 & 0.9165 & 0.0584 & -0.0824 & & 53.90 & 0.9450 & 0.8982 & 0.2747 & -0.0385 \\
\hline & 46.85 & 0.8935 & 0.9147 & 0.1108 & -0.0696 & & 53.95 & 0.9023 & 0.7597 & 0.4179 & 0.0073 \\
\hline & 46.95 & 0.8702 & 0.8656 & 0.2319 & -0.0262 & & 54.00 & 0.9388 & 0.8848 & 0.2289 & -0.0257 \\
\hline & 47.05 & 0.8003 & 0.8133 & 0.2310 & -0.0141 & & 54.05 & 0.9427 & 0.8675 & 0.3101 & -0.0236 \\
\hline & 47.10 & 0.9064 & 0.9083 & 0.1830 & -0.0230 & & 54.10 & 0.9569 & 0.8738 & 0.2443 & -0.0436 \\
\hline & 47.15 & 0.8740 & 0.8809 & 0.1131 & -0.0474 & & 55.25 & 0.9023 & 0.8025 & 0.2484 & 0.0337 \\
\hline & 47.20 & 0.8642 & 0.8902 & 0.1262 & -0.0661 & & 55.30 & 0.8605 & 0.8198 & 0.2426 & -0.0075 \\
\hline & 47.25 & 0.9192 & 0.8958 & 0.1330 & -0.0611 & & 55.35 & 0.9070 & 0.8517 & 0.2399 & -0.0136 \\
\hline & 47.30 & 0.9024 & 0.8687 & 0.2236 & -0.0555 & & 55.40 & 0.9241 & 0.8391 & 0.3827 & -0.0130 \\
\hline & 47.35 & 0.8993 & 0.8709 & 0.2267 & -0.0226 & & 55.45 & 0.9384 & 0.8627 & 0.2866 & -0.0308 \\
\hline & 47.40 & 0.8691 & 0.8568 & 0.1914 & -0.0274 & & 55.50 & 0.9411 & 0.9038 & 0.2795 & -0.0457 \\
\hline & 47.45 & 0.9068 & 0.9010 & 0.1764 & -0.0637 & & 55.55 & 0.9240 & 0.8252 & 0.4293 & -0.0227 \\
\hline & 47.50 & 0.9042 & 0.8908 & 0.1245 & -0.0691 & & 55.60 & 0.9385 & 0.8952 & 0.2045 & -0.0472 \\
\hline \multirow[t]{3}{*}{ Site 849: } & & & & & & \multirow{3}{*}{\multicolumn{2}{|c|}{$\begin{array}{r}\text { Note: } \text { Comm. } \\
=\text { west Pac } \\
\text { assemblage }\end{array}$}} & ality $\mathbf{T}$ & $-\operatorname{tran}$ & assembl & - West B \\
\hline & 143.20 & 0.8123 & 0.4374 & 0.6303 & 0.1352 & & & & & & \\
\hline & 143.40 & 0.9066 & 0.7171 & 0.5016 & 0.0482 & & & & & & \\
\hline
\end{tabular}

